\title{
Peroxide Based Organic Explosives
}

\section{Lemi Türker}

Department of Chemistry, Middle East Technical University, Üniversiteler, Eskişehir Yolu No: 1, 06800 Çankaya/Ankara, Turkey; e-mail: lturker@gmail.com; lturker@metu.edu.tr

\begin{abstract}
In recent years progressively increasing terrorist activities, which use homemade explosives; such as acetone peroxide and other cyclic organic peroxides have led to worldwide awareness by security and defense agencies. Then the development of methodologies for the detection of cyclic organic peroxides have become an urgent need. Until quite recently, most of the current technology in use for trace detection of explosives had been unable to detect these energetic compounds. Differences in physical properties between cyclic organic peroxides is the main barrier for the development of a general method for analysis and detection of the peroxide explosives. In this short review, the most relevant contributions related to preparation, characterization and detection of the most important cyclic organic peroxides have been presented. It also includes few recent investigations about the toxicity and metabolism of some peroxide explosives.
\end{abstract}

\section{Introduction}

Organic peroxides are another class of explosives which have been the focus of interest of many researchers in recent years [1,2]. Cyclic organic peroxides-based homemade explosives have been used to build weapons of mass destruction in the Middle East for several years. Increased terrorist activities, which use acetone peroxide and other cyclic organic peroxides as homemade explosives, have led to worldwide awareness by security and defense agencies. Development of methodologies for the detection of triacetone triperoxide (TATP), hexamethylene triperoxide diamine (HMTD), tetramethylene diperoxide dicarbamide (TMDD) and other cyclic organic

Received: July 24, 2021; Accepted: August 27, 2021

Keywords and phrases: peroxide explosives, TATP, HMTD, TMDD, detection.

Copyright (C) 2021 Lemi Türker. This is an open access article distributed under the Creative Commons Attribution License, which permits unrestricted use, distribution, and reproduction in any medium, provided the original work is properly cited. 
peroxides have become an urgent priority [3]. Although hydrogen peroxide (HP) was discovered nearly two hundred years ago its special properties, present in concentrated solutions, started to be utilized just before World War II, by German researchers. The detonable properties of concentrated solutions of hydrogen peroxide were discovered at that time as well. The mixtures researched so far have exhibited relatively low nitrogen oxides emissions as well as sufficient explosive power, stability, sensitivity and versatility. Additionally, HP based explosives can be prepared in situ which eliminates the logistical difficulties usually encountered in shipping and storing high explosives. Both, the fuel component (liquid or in the form of loose material, optionally with the additional activator) and a solution of specially stabilized HP (the liquid phase), can remain in long term non-explosive storage, until required. However, when mixed, the resulting mixture becomes a detonator (or detonator-booster) sensitive high explosive with high gas volume, high bulk energy and consistent detonation velocity (quite appropriate for the mining industry). The mixtures investigated so far appeared to be relatively easy to use and quite adaptable [4].

\section{Energetic Organic Peroxides}

Although, peroxides are inherently unstable, not all organic peroxides can be used as explosives. Some organic peroxides are regarded as explosives and are able to sustain a powerful detonation without added fuel. An important distinction is to be made between organic peroxides and explosive mixtures containing hydrogen peroxide. Hydrogen peroxide-organic matter (HPOM) mixtures contain hydrogen peroxide as the oxidizer and the fuel can be almost any finely ground organic material, although performance will vary based on the fuel used. Since, a HPOM contains no covalent bonds between the peroxide unit and organic moieties it is not considered an organic peroxide. HPOMs are significantly less friction sensitive than organic peroxide explosives (OPEs), and usually less powerful [5]. The most commonly encountered OPEs include TATP, HMTD and methyl ethyl ketone peroxide (MEKP). Under the title MEKP, a family of cyclic and open-chain organic peroxide oligomers (formed in the reaction of methyl ethyl ketone and hydrogen peroxide) reside [6].

Peroxides are a class of substances which contain an O-O single bond. They have two subclasses: inorganic and organic peroxides. Inorganic peroxides are metal salts which possess the peroxide anion $\mathrm{O}_{2}{ }^{2-}$, such as $\mathrm{BaO}_{2}$ or $\mathrm{Na}_{2} \mathrm{O}_{2}$, salts of Caro's acid 
$\mathrm{H}_{2} \mathrm{SO}_{5}$ and hydrogen peroxide. Many decades barium peroxide was used for the industrial preparation of oxygen and also hydrogen peroxide. Nova days, better ways of manufacturing have been employed based on the Linde process for oxygen, respectively the antrachinone process for hydrogen peroxide production [7]. Inorganic peroxides have found many applications. For instance, in combination with magnesium, $\mathrm{BaO}_{2}$ was also used in ignition pellets for thermite mixtures [7]. In pyrotechnical formulations $\mathrm{BaO}_{2}$ is used as oxygen delivery agent and, like the other barium salts, produces a green flame color. Hydrogen peroxide itself found application in the Aggregate 4 (V2)-rocket as steam producing agent to run the fuel pump of the rocket engine [8] and as the so called T-Stoff, [9] in combination with Z-Stoff (a solution of calcium permanganate or potassium permanganate in water) [9] for start assistance devices of the Fieseler Fi 103 (V1) missile, the Messerschmitt Me 321 glider and the Arador AR 234 operational bomber [10]. Moreover it was again used as T-Stoff in the Messerschmitt Me 163 [10], the first plane to exceed Mach 1, this time in combination with C-Stoff, a mixture of methanol, hydrazine-hydrate and water [9]. However, in the following sections the term "peroxide" always means "organic peroxide". Figure 1 shows some of subclasses of organic peroxides.<smiles>[R]OO[R7]</smiles>

Peroxyethers

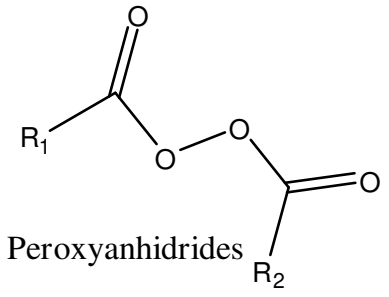

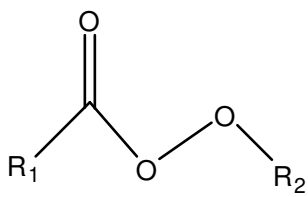

Peroxyesters

\section{$\mathrm{ROOH}$}

Hydroperoxides

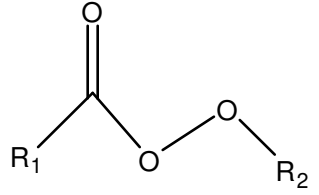

Peroxyacids

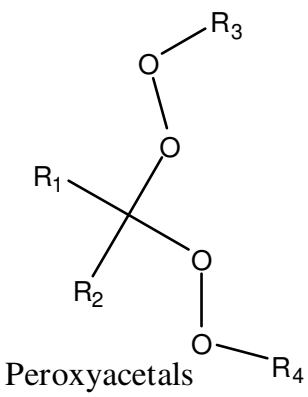

Figure 1. Some organic peroxides.

Organic peroxides are a relatively unexplored class of substances. The most prominent example is acetone peroxide which was synthesized for the first time by the German chemist Richard Wolffenstein in 1895 [11]. His intention was to oxidize Coniin 
with hydrogen peroxide and used acetone as solvent. The white precipitate formed exhibited to have explosive properties. Detailed investigations concerning the composition of the product were performed later and it was found that the main fraction is a trimeric molecule (triacetone tri peroxide, TATP). The trimer is accompanied by a dimer (diacetone di peroxide, DADP) [12]. The ratio of the two possible products are formed depends on the reaction conditions (mostly the concentration of the acid) used during the reaction. Generally concentrated and strong acids favor the formation of DADP whereas lower acid concentrations or acid free conditions lead to the formation of TATP. Under the catalysis of tin (IV), a tetrameric form can be isolated [13]. Although, Wolffenstein patented his invention his attempts to sell it to the German army failed, because the high sensitivity and especially the high volatility of the compound. In recent years, cocrystallization with trinitro-trihalogeno-benzenes was found to be an effective way to reduce the sensitivity of DADP. Unfortunately, however, this strategy turned out not successful for TATP, because the $\mathrm{O}$-atoms are not accessible in the trimeric structure $[14,15]$. Hexamethylene triperoxide diamine (HMTD) was discovered in the same period as acetone peroxide by Ludwig Leger from the reaction of ammonia and Lampensäure. This compound has been found to be also sensitive towards shock and friction and shows a fast DDT which means it can be used as a primary explosive. Also, it showed less sensitivity than the primary explosives used before. Therefore, it found application in initiation charges for mining operations for a short period of time. Like acetone peroxide it is prepared in a straightforward way today by reacting hydrogen peroxide with hexamine and citric acid. The unpredictable behavior due to the, for modern standards, too high sensitivity to all external stimuli is again the most significant drawback of HMTD. Methyl ethyl ketone peroxide is a liquid peroxide which is similarly prepared from the ketone and hydrogen peroxide under acidic conditions [16]. It consists of a mixture of several different molecules, but an open chain dimer is the main product. Figure 2 shows the molecular structures of DADP, TATP, TrATrP, HMTD and MEKP.

For most peroxides, the general decomposition mechanism is not investigated in detail until now [17]. Only for acetone peroxide deeper studies have been performed. In this case, the main reaction products are carbon dioxide, methane, ethane, ethylene and acetone and no significant amount of heat is released [18]. Under a nitrogen atmosphere, no flame can be observed [18] (entropic detonation).

A notable characteristic of OPEs is one of the reasons that they have no use in commercial or military purposes. It is because of their very high sensitivity to 
mechanical impact, friction, heat and electrostatic discharge. TATP, HMTD and MEKP are all extremely sensitive to such initiation stimuli. Although, OPEs, are extremely dangerous to handle, they occupy a unique class in the explosive world by being both easily accessible and sensitive. The sensitiveness of TATP makes it a prime candidate for converting a source of heat or spark (i.e. electrical current) into a detonation wave which is capable of initiating a larger bulk explosive such as ANFO.

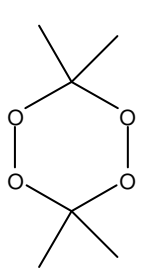

DADP

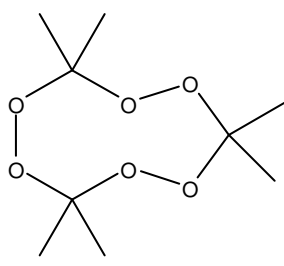

TATP

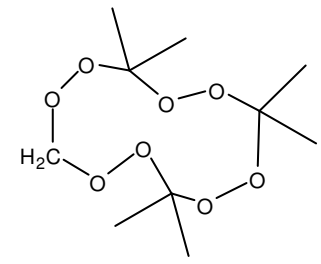

$\operatorname{Tr} \mathrm{ATrP}$

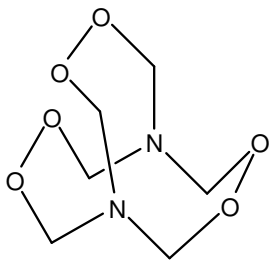

HMTD

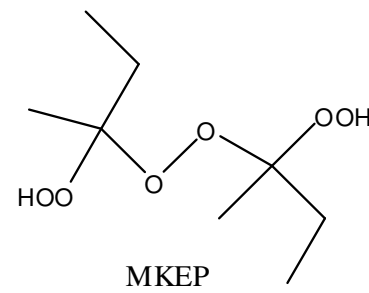

MKEP

Figure 2. Structural formulas of DADP, TATP, TrATrP, HMTD and MEKP.

\section{Synthesis}

$\mathrm{H}_{2} \mathrm{O}_{2}$ which is frequently used as an oxidizing agent in organic chemistry converts certain class of materials in to the peroxide derivatives without destructive oxidation.

Ketones or ketals are readily converted into the corresponding gem-dihydroperoxides in high yields by treatment with ethereal $\mathrm{H}_{2} \mathrm{O}_{2}$ at ambient temperatures in the presence of 2-5 mol \% phosphomolybdic acid (PMA) as catalyst [19].

In a work published in 2016, the synthesis, structure, and energetic materials properties of a series of aromatic peroxy acid compounds are described [20]. Benzene1,3,5-tris(carboperoxoic) acid is a highly sensitive primary energetic material, which possesses impact and friction sensitivities similar to those of triacetone triperoxide (TATP). By contrast, benzene-1,4-bis(carboperoxoic) acid, 4-nitrobenzoperoxoic acid, and 3,5-dinitro benzoperoxoic acid are known as having much less sensitive, with impact 
and friction sensitivities, close to those of the secondary energetic material such as 2,4,6trinitrotoluene. Additionally, the calculated detonation velocities of 3,5dinitrobenzoperoxoic acid and 2,4,6-trinitrobenzoperoxoic acid far exceed that of 2,4,6trinitrotoluene. The solid-state structure of 3,5-dinitrobenzoperoxoic acid contains intermolecular $\mathrm{O}-\mathrm{H} \cdots \mathrm{O}$ hydrogen bonds and numerous $\mathrm{N} \cdots \mathrm{O}, \mathrm{C} \cdots \mathrm{O}$, and $\mathrm{O} \cdots \mathrm{O}$ close contacts. These attractive lattice interactions may be responsible for the less sensitive nature of 3,5-dinitrobenzoperoxoic acid [20].

A $\mathrm{Cu}^{\mathrm{I}}$-catalyzed oxidation of enynes using $\mathrm{N}$-hydroxybenzotriazole $(\mathrm{HOBt})$ or $\mathrm{N}$ hydroxyphthalimide (NHPI) with molecular oxygen has been developed to yield propargyl hydroperoxides [21]. The reaction occurs under $\mathrm{O}_{2}$ at $0^{\circ} \mathrm{C}$ to afford hydroperoxides in 1-4 h. This method provides an alternative to $\mathrm{H}_{2} \mathrm{O}_{2}$ or $\mathrm{tBuOOH}$ by using molecular oxygen as the source of oxygen atoms. The resulting hydroperoxides can be protected prior to purification and converted into alkoxyamines upon treatment with hydrazine [21].

Jiang et al., observed that $\mathrm{SnCl}_{4} \cdot 5 \mathrm{H}_{2} \mathrm{O}$ and $\mathrm{SnCl}_{2} \cdot 2 \mathrm{H}_{2} \mathrm{O}$ were efficient catalysts for the oxidation of acetone with $30 \%$ hydrogen peroxide at room temperature. The reaction produces tetrameric acetone peroxide which was fully identified by molecular weight determination, elemental analysis, FTIR, NMR and MS [22].

Acetone cyclic triperoxide (ACTP) was produced over titania-incorporated mesoporous material catalysts from a mixture of acetone and hydrogen peroxide under a mild and acid-free condition [23]. Formation of ACTP was confirmed by single-crystal X-ray crystallography. The formation of ACTP could be observed in this study because of using the catalyst with a large amount of titania-incorporation at $23 \mathrm{wt} . \%$ and its large pore size [23].

In another study, $\operatorname{Re}_{2} \mathrm{O}_{7}$ in $\mathrm{CH}_{3} \mathrm{CN}$ has been shown to be a remarkably efficient and mild catalyst for the peroxyacetalization of ketones, aldehydes, or acetals by $\mathrm{H}_{2} \mathrm{O}_{2}$ to generate 1,1-dihydroperoxides. $\mathrm{Me}_{3} \mathrm{SiOReO}_{3}$ and methyl rhenium trioxide (MTO) are also effective catalysts under these reaction conditions [24].

A novel method used to synthesize diacetone diperoxide (DADP) from acetone and hydrogen peroxide without the presence of a catalytic agent has been reported [25]. Previously reported syntheses used toluenesulfonic and $m$-sulfonic acid as catalyst. By this method DADP was prepared with a purity of $99.99 \%$. It was found that the melting 
point range $\left(128.5-134.5^{\circ} \mathrm{C}\right)$ was consistent with the reported values. The success of the reported procedure depends on controlling the ratio between acetone and hydrogen peroxide as well as the temperature of the reaction mixture. The purified crystalline DADP samples were characterized by means of NMR analysis, Raman, and IR spectroscopy, differential scanning calorimetry, gas chromatography and open atmosphere chemical ionization mass spectrometry. The structure was compared to the well-known cyclic trimer triacetone triperoxide (TATP). The proposed synthetic scheme can be useful while preparing small amounts of the cyclic organic peroxide for characterization and fundamental studies and for formulation of standards of gas chromatography, high performance liquid chromatography, and mass spectrometry [25].

\section{Decomposition and Other Reactions}

Yaremenko et al., extensively search the literature of peroxides [26]. Their review was the first to collate and summarize main data on various named and unnamed rearrangement reactions of peroxides. They have noted that in the chemistry of peroxides two types of processes are considered under the term rearrangements. These types are conventional rearrangements occurring with the retention of the molecular weight and transformations of one of the peroxide moieties after O-O-bond cleavage. Detailed information about the well known Baeyer-Villiger, Criegee, Hock, KornblumDeLaMare, Dakin, Elbs, Schenck, Smith, Wieland, and Story reactions has been given. Unnamed rearrangements of organic peroxides and related processes are also analyzed. The rearrangements and related processes of important natural and synthetic peroxides have been discussed separately.

Their review was the first to offer a complex analysis of the available data on rearrangements of peroxides published in the last 15-20 years as well as mentioning the history of the discovery of particular reactions and transformations [26]. The rearrangements and related processes have been classified according to the type of the catalysts used including the acid- and base-catalyzed processes, reactions catalyzed by variable-valence metals, photochemical and thermal action. In the article a special emphasis is drawn to current trends in the performance and application of rearrangements of organic peroxides, such as organocatalysis, asymmetric synthesis, and the use of transition metal-peroxo complexes for the preparation of compounds interesting for various pharmacological applications. The published data have been 
summarized in the review, for the first time, providing an insight into the common and different features of the reaction mechanisms. It allows predicting experimental and structural requirements for performing rearrangements with specified results. An analysis performed on the published data have shown that there are numerous new and unnamed processes related to name reactions. The development and investigation of these processes are apparently the future of peroxide chemistry [26].

Bali et. al. have noted that research to counter the threat of organic peroxides such as triacetone triperoxide (TATP) is at times hampered by their inherent extreme sensitiveness and volatility [27]. Their work describes an approach to lowering some risks associated with the handling of TATP in the laboratory through the use of an analog species, tripentanone triperoxide (TPTP). In their study sensitiveness has been tested via standard methods. GCMS analysis performed has shown that TPTP degrades via similar mechanisms to TATP under a range of conditions. Slight differences in product composition were traced to side reactions which may also affect impurities present in homemade TATP synthesis. Also, a pilot field trial was conducted to evaluate TPTP as a substitute for TATP for the purpose of explosive detection dog (EDD) scent training. The degradation studies have provided some insights into the complexities of the acidic degradation of cyclic peroxides with potential forensic application, and TPTP's inadequacy as a TATP pseudoscent is a valuable example of the limitations of such training aids [27].

Pioneers of the field faced extraordinary risks when dealing with organic peroxides such as TATP due to the extreme sensitivity of this class of explosives, and to a lack of a robust chemical means of safe and rapid neutralization. Bali et al., studied degradation reactions of some peroxide compounds [28]. The Lewis acids $\mathrm{TiCl}_{4}$ and $\mathrm{SbCl}_{3}$ have been found to demonstrate a novel degradation mechanism. $\mathrm{TiCl}_{4}$ degrades cyclic peroxide in minutes when used in a two-fold excess, or $\sim 3$ hours at half equivalence. Furthermore, the products cannot re-form peroxide compounds as is the case with acid degradation, suggesting that the two mechanisms are fundamentally different. The Lewis acids mediate a rearrangement reaction in the cyclic peroxide backbone leading to relatively innocuous products through deactivation of oxidizing $\mathrm{O}$. On the other hand, substoichiometric $\mathrm{TiCl}_{4}$ reactions highlight a secondary reaction pathway that also leads to some oxidative chlorination products. They are possibly mediated by an unconfirmed titanium-oxychloride species. The experiments showed that $\mathrm{SbCl}_{3}$ exhibits similar 
reactivity to $\mathrm{TiCl}_{4}$, although at a slower rate. In the article also a mechanism is proposed, consistent with the observations for both stoichiometric and sub-stoichiometric quantities of $\mathrm{TiCl}_{4}[28]$.

The thermal decomposition of two peroxides, triacetone triperoxide (TATP) and hexamethylene triperoxide diamine (HMTD), was investigated as a function of pressure, temperature and atmosphere by using T-jump/FT-IR spectroscopy [29]. In an argon atmosphere at fast heating rates, the dominant decomposition product of TATP was found to be methane rather than acetone which dominates at slow heating rates. The products of TATP were different in argon and in air which implies that decomposition occurs primarily in the gas phase. Whereas, the products from HMTD were independent of the surrounding atmosphere implying that it decomposes primarily in the condensed phase. Furthermore the experiments revealed that the IR spectra of the decomposition products of HMTD exhibited some dependence on pressure but not on the pyrolysis filament temperature [29].

The previously reported computational study of the decomposition pathways of triacetone triperoxide (TATP), predicted that unlike most energetic materials, which involve self-combustion of fuel and oxidants, TATP decomposes via a thermoneutral, non-redox pathway that involves entropy burst. TATP is a highly sensitive explosive, which can be initiated by minor thermal or mechanical stress, including open flame, hot surface, electrostatic discharge, impact, friction, etc. The resultant event, either deflagration or detonation, is usually accompanied by the formation of a fireball. In the study, time-resolved monitoring of deflagration or detonation of TATP was studied using a fast video camera following initiation by a short pulse focused laser beam. While a fireball always accompanies the explosion of TATP under air, the formation of a fireball is totally prevented under a nitrogen atmosphere. These observations indicate that combustion of the gaseous primary products occurs as a secondary event only in the presence of exogenous oxygen. The composition of the product mixture was found to depend on the experimental conditions. With long pulse focused laser beam (150 $\mu$ s at $1064 \mathrm{~nm}$ ) at either 210 or $110 \mathrm{~mJ}$, the small amounts of charcoal needed for initiation suggest that the energy required to initiate TATP by pulse laser is $4-10 \mathrm{~mJ}$, much smaller than the energy required for initiation by either mechanical stress or electric discharge. This time-resolved study highlights the very unusual properties of the peroxide based explosives [18]. 
The group had previously reported calculations predicted that each molecule of TATP, would undergo a multistep decomposition pathway to produce three molecules of acetone and one molecule of ozone. This seems to be the case under conditions of slow decomposition, where acetone is indeed produced in high proportions. In contrast, under more drastic conditions, which lead to fast decomposition rates, acetone could undergo further fragmentation. In the $\mathrm{T}$-jump experiments the reported time frame for decomposition was $0.1-20 \mathrm{~s}$ with temperatures increasing at a rate of $2000{ }^{\circ} \mathrm{C} / \mathrm{s}$. In their laser initiation experiments the time frame was 10-2-10-3 s and 10-5-10-6 for deflagration and detonation, respectively. Although the temperature of these events was not measured it is known that detonation processes produce much higher temperatures than deflagration. Pyrolysis of acetone is known to produce methane, ethane and ketene, and the latter can undergo further reactions to produce ethylene. Accordingly, the observed detonation and deflagration products, including carbon dioxide, could be explained by pyrolysis of acetone [18].

Sinditskii et al., considered two potentially initiating explosive peroxides, diacetonediperoxide (DADP) and triacetonetriperoxide (TATP), and studied their thermochemical properties. In order to get the internally self-consistent estimations of gas-phase enthalpy of formation of DADP and TATP, their values were calculated by combining Gaussian-4 (G4) theory with an isodesmic reaction scheme. The energies of combustion $(\Delta \mathrm{cU})$ were measured and the standard enthalpies of formation $\left(\Delta \mathrm{fH} 298^{\circ}\right)$ of DADP and TATP were derived using the standard enthalpies of formation of the combustion products. Also the heat of explosion was measured for small low-pressed charges of the peroxides. The obtained enthalpies of formation of DADP and TATP were found to agree well with quantum chemical calculations. The results reasonably account for the observed derivative parameters, namely heats of decomposition, combustion, and detonation [30].

Chemicals possessing multiple peroxide functionalities in their structures, such as triacetone triperoxide (TATP), diacetone diperoxide (DADP), or hexamethylene triperoxide diamine (HMTD), can explode. They are impractical and are not used by legitimate military groups because they are shock and heat sensitive compared to military explosives. They are attractive to terrorists because synthesis is straightforward, requiring only a few easily obtained ingredients. Oxley et al., in their paper discuss methods to degrade peroxide explosives, DADP, TATP, and HMTD, chemically, at 
room temperature. A number of mixtures containing metals (e.g., zinc, copper) and metal salts (e.g., zinc sulfate, copper chloride) were found to be effective, so that some capable of destroying TATP solutions in a few hours. Strong acids proved useful against solid peroxide materials. On a $1 \mathrm{~g}$ scale, however addition of concentrated sulfuric acid caused TATP to detonate. Thus, this technique should only be used to destroy small-laboratory quantities [31].

This study demonstrated that strong acids, such as methyl sulfonic acid (99\%), sulfuric acid (98\%), and nitric acid (70\%), when applied directly to solid (5 mg) TATP, DADP, and HMTD, even without stirring, accomplished destruction in $15 \mathrm{~min}$. However, this destruction technique seemed to be unsuitable for use even in gram quantities of TATP. The authors believed that the reaction with acid is exothermic. However, this is not noticeable on the milligram-scale, but it provides sufficient energy to initiate the TATP on the gram-scale. They did not performed gram-scale tests on DADP or HMTD.

The thermal decomposition of triacetone triperoxide (TATP) was investigated over the temperature range 151 to $230{ }^{\circ} \mathrm{C}$ by Oxley et al., and found to be first order out to a high degree of conversion [32]. Arrhenius parameters were calculated yielding activation energy of $151 \mathrm{~kJ} / \mathrm{mol}$ and pre-exponential factor, $3.75 \times 10^{13} \mathrm{~s}^{-1}$. Under all conditions the principle decomposition products were acetone (about 2 mole per mole TATP in the gasphase and $2.5 \pm 2.6$ mole per mole in condensed-phase) and carbon dioxide. Minor products included some molecules ascribed to reactions of methyl radical namely ethane, methanol, 2-butanone, ethyl acetate which increased at high temperature. Methyl acetate and acetic acid were also formed in the decomposition of neat TATP; the former was more evident in the gas phase decompositions $\left(151^{\circ} \mathrm{C}\right.$ and $\left.230^{\circ} \mathrm{C}\right)$ and the latter in the condensed-phase decompositions $\left(151^{\circ} \mathrm{C}\right)$. The decomposition of TATP in condensedphase or in hydrogen-donating solvents enhanced acetone production, suppressed $\mathrm{CO}_{2}$ production, and slightly increased the rate constant (a factor of $2 \pm 3$ ). All observations were interpreted in terms of decomposition pathways initiated by homolysis of peroxide bond(s) [32]. Although the products and activation energies for TATP decomposition are similar to those of DADP, the authors do not believe the decomposition of TATP proceeds through the same intermediate diradical as DADP because no DADP was observed in partially decomposed TATP. Furthermore, neat TATP decomposed significantly faster than DADP. Had DADP formed, it should have been sufficiently 
long-lived for observation. Following $\mathrm{O}-\mathrm{O}$ bond homolysis rapid $\mathrm{CO}$ scission yields two molecules of acetone. The fate of the remaining atoms depends on the reaction conditions. Gas phase favors the formation of dimethyl dioxirane; high temperature favors its decomposition to carbon dioxide; lower temperature favors production of methyl acetate. Condensed-phase or reaction in a hydrogen donating solvent favors the formation of a third molecule of acetone rather than dioxirane.

On the other hand, Dubnikova et al., studied thermal decomposition pathway of TATP and DADP [33]. Both X-ray crystallography and electronic structure calculations using the cc-pVDZ basis set at the DFT B3LYP level were employed to study the explosive properties of triacetone triperoxide (TATP) and diacetone diperoxide (DADP). The thermal decomposition pathway of TATP was investigated by a series of calculations that identified transition states, intermediates, and the final products. Counter intuitively, these calculations predict that the explosion of TATP is not a thermo chemically highly favored event. It rather involves entropy burst, which is the result of formation of one ozone and three acetone molecules from every molecule of TATP in the solid state [33].

The kinetics and stoichiometry of the formation of active oxygen (AO) in the acidic decomposition of trimeric (TATP) and dimeric (DADP) cyclic acetone peroxides are considered. Fe(III) produced as a result of $\mathrm{Fe}$ (II) oxidation with active oxygen has been determined using rhodanide procedure. The kinetics of the formation of active oxygen is described by a first order equation. The effective rate constant of TATP decomposition depends on the Hammett acidity function $\mathrm{H}_{0}: \log \mathrm{k}_{\text {eff }}=-\mathrm{H}_{0}-2.6\left(\mathrm{k}_{\text {eff }}\right.$ is in $\left.\mathrm{s}^{-1}\right)$. Consequently, the decomposition rate of TATP is limited by protonation. In the $\mathrm{HCl}$ and $\mathrm{H}_{2} \mathrm{SO}_{4}$ concentration range from 0.006 to $2.9 \mathrm{~mol} / \mathrm{L}$, the decomposition of DADP occurs with $\mathrm{k}_{\mathrm{eff}}=0.0010 \pm 0003 \mathrm{~s}^{-1}$ at a $\mathrm{Fe}(\mathrm{II})$ concentration of $3.5 \mathrm{mmol} / \mathrm{L}$ and $\mathrm{k}_{\text {eff }}$ depends linearly on the concentration of $\mathrm{Fe}(\mathrm{II})$ [34].

The initial stage of the acidic decomposition of TATP can be represented as follows:

$$
\begin{gathered}
\text { TATP }+\mathrm{H}_{3} \mathrm{O}^{+} \leftrightarrow \text { TATP } \cdot \mathrm{H}_{3} \mathrm{O}^{+}, \\
\text {TATP } \cdot \mathrm{H}_{3} \mathrm{O}^{+} \rightarrow \text { TATP } \cdot \mathrm{H}^{+}+\mathrm{H}_{2} \mathrm{O},
\end{gathered}
$$

TATP $\cdot \mathrm{H}^{+} \rightarrow$ ring opening and AO formation

On the other hand, the results obtained for DADP are evidence that it is impossible 
to decompose it rapidly into $\mathrm{H}_{2} \mathrm{O}_{2}$ and acetone in the presence of a heterogeneous acid catalyst similar to the catalyst suggested for the decomposition of TATP. However, $\mathrm{Fe}(\mathrm{II}) \mathrm{can}$ be used to activate the acidic decomposition of DADP.

\section{Explosive Characters and Usage}

Triacetone triperoxide (TATP) is easily formed from acetone in sulfuric acid solution when acted upon hydrogen peroxide. Like most other organic peroxides TATP also has very high impact, friction and thermal sensitivity. Thus, TATP displays the properties of a primary explosive. For this reason and because of its tendency to sublime it is not used in practice (apart from terrorist and illegal activities). Because of the use of TATP by terrorists, a reliable and fast detection of this material is desirable. In addition to conventional analytical methods such as mass spectrometry and UV (ultra-violet) spectroscopy, specially trained explosive-detection dogs (EDD) play an important role in the detection of organic peroxides. Klapötke, and Wloka discussed typical organic peroxides so-called homemade explosives (HMEs) (which have or may be used by terrorists) including TATP (triacetone triperoxide), HMTD (hexamethylene triperoxide diamine), MEKP (methyl ethyl ketone peroxide) and DADP (diacetone diperoxide) [35].

\section{Triacetone Triperoxide (TATP)}

TATP is an organic peroxide that acts as a high explosive. It is highly susceptible to heat, friction and shock. Because of its instability, it has been called the "Mother of Satan". The explosion of TATP does not generate much energy. In conventional high explosives such as TNT, each molecule contains intramolecularly both a fuel component and an oxidizing component. When the explosive detonates, the fuel part is oxidized, and as this combustion reaction spreads, it releases large amounts of heat. Whereas, the explosion of TATP involves entropy burst, causing the formation of one ozone and three acetone molecules from every molecule of TATP in the solid state. Hence, a small amount (such as a few hundred grams) of the material produces hundreds liters of gas in a fraction of a second. TATP can be prepared easily in a so-called basement lab using commercially available starting materials easily obtained from hardware stores, pharmacies and stores selling cosmetics. TATP is a fairly easy explosive to make, as far as explosives manufacturing goes. All it requires is acetone, concentrated hydrogen peroxide (perhydrol) and a strong acid like hydrochloric or preferably sulfuric acid. In the near past, TATP was used as the explosive in the July 2005 London bombings, a 
series of coordinated terrorist bomb blasts that hit London's public transport system. Richard Reid, who attempted to down American Airlines Flight 63 with a bomb concealed in his shoe, employed a device containing plastic explosives with a TATP trigger [36,37].

\section{Methyl Ethyl Ketone Peroxide (MEKP)}

MEKP is an organic peroxide too, a highly explosive material similar to TATP. However, it is very dangerous to prepare. MEKP is a colorless, oily liquid at room temperature and pressure, while TATP is a white solid. It's slightly less sensitive to shock and temperature than TATP and more stable in storage. It is prepared from methyl ethyl ketone (MEK) and hydrogen peroxide. Its diluted solutions are used in industry and by hobbyists as the catalyst that initiates the polymerization of polyester resins used in glass-reinforced plastic and casting. MEKP does this through the production of free radicals. It has been reported that MEKP might be blamed for being used in the alleged 2006 transatlantic aircraft plot to destroy planes flying from the United Kingdom to the United States. TATP remains another reported possibility [38].

\section{Hexamethylene Triperoxide Diamine (HMTD)}

HMTD is a high-explosive organic peroxide compound. It was first synthesized in 1885. The theorized structure lends itself well to acting as an initiating, or primary, explosive. In spite of the fact that it was still quite sensitive to shock and friction, it was relatively stable compared to other initiating explosives of the time, such as mercury fulminate. HMTD proved to be relatively inexpensive and easy to synthesize. Despite no longer being used in any official application, it remains a fairly popular HME and has been used in a large number of suicide bombings throughout the world. It remains as a possible agent used in the July 2005 London bombings. Like other organic peroxides (such as TATP) HMTD is an unstable compound sensitive to shock, friction and heat. It also reacts with most common metals, which can lead to detonation. This makes the substance extremely dangerous to manufacture. However, HMTD is very stable when it is acid-free and does not quickly sublimate like its acetone counterparts [39].

The power of various explosive mixtures based on triacetone triperoxide (3,3,6,6,9,9-hexamethyl-1,2,4,5,7,8-hexoxonane, TATP), ammonium nitrate (AN), urea nitrate (UrN) and water (W), namely TATP/AN, oil/AN, TATP/UrN, TATP/W and TATP/AN/W, was studied using the ballistic mortar test. The ternary mixtures of 
TATP/AN/W have relatively high power in case of the low water contents. Their power decrease significantly with increasing the water content in the mixture to more than $30 \%$ [40].

Triacetone triperoxide (TATP), diacetone diperoxide (DADP) and hexamethylene triperoxide diamine (HMTD) (which are sensitive and powerful organic peroxide explosives) have been encountered by the UK Police, both in terrorist and non-terrorist cases. Initially, the recovery of a substantial quantity of TATP in the UK revealed that there was a lack of reliable chemical, explosive and analytical data on organic peroxide explosives. Since then, extensive research has been carried out in order to build up a detailed forensic knowledge of these materials. A systematic study of the physical and chemical properties of these explosives has been undertaken along with methods for qualitative analysis and detection [41]. The research carried out has found TATP, DADP and HMTD to be powerful primary explosives. They can be prepared in the home starting from readily available raw materials. However, their sensitivity to accidental initiation makes them extremely hazardous materials to handle. They burn rapidly or instantaneously when exposed to a naked flame. Analytical techniques including FT-IR, TLC, GC/MS and LC/MS can be used for their forensic analysis and identification [41].

Araos and Onederra studied the detonation process of novel hydrogen peroxidebased explosives using high speed video [42]. In order to characterize non-ideal explosives, the explosives industry rely on unconfined velocity of detonation tests and underwater pond tests (where possible). Although, the information obtained from such tests is quite helpful, it cannot give much information about the dynamics of the detonation reaction process. The detonation process has been studied for ideal explosives and the reaction zone length have been measured. However, for non-ideal explosives, that type of information is not available. Therefore, the primary aim of work by Araos and Onederra was to capture the detonation process of a non-ideal explosive using high speed video camera to determine the zone length.

As part of a project to develop alternative explosive formulations, it was decided to investigate the detonation process of these new AN-free explosive mixtures that use hydrogen peroxide instead of AN as the oxidizer. The detonation performance of these mixtures was quantified by direct VOD measurements but also supported by high speed video. A Photron SA-2 camera was used for the tests at speeds between 50,000 and 100,000 frames per second. 
High speed video footage allowed the authors to observe the dynamics of the detonation process closely. Also it allows to measure aspects associated with the size of the reaction region when different variables were changed, including density, diameter of the charge, type of void sensitization; and geometry of the explosive charge (e.g. cylindrical vs. rectangular).

In the project, in addition to observations of the reaction regions, other interesting characteristics of the detonation process were observed and analyzed which included the color of the expanding gases (which can be related to temperature) for different types of sensitization; as well as the angle of the expanding gases with respect to the charge axis. The transition between detonation to failure was also observed [42].

An interesting study has been presented by Dobson who showed in his thesis that TATP could be decomposed or even detonated through a controlled ultrasonication process [43]. Either outcome could be used by law enforcement officials as the basis for a standoff procedure for the destruction of bulk quantities of TATP. The results indicated that the shockwave and pressure and temperature extremes caused by ultrasonication of a medium could exploit TATP's sensitivity to shock, friction, heat and electrostatic charge and induce decomposition of the molecule in a controlled (i.e. distant) environment [43].

The work by Onederra and Araos reports the results of a preliminary monitoring program designed to quantify the in situ performance of newly developed hydrogen peroxide based explosives. Their advantage is that they do not produce NOx fumes upon detonation. Direct measurements of velocity of detonation, pressure, temperature and displacement were obtained. Detonation velocities ranged from 2607 to $5100 \mathrm{~m} \mathrm{~s}^{-1}$ with differences mainly attributed to density variations. The data showed consistent relative differences in pressure and temperature with measurements in the range of $0.6-2.6 \mathrm{GPa}$ and $2036-3551{ }^{\circ} \mathrm{C}$, respectively. Multiple hole tests showed that there was no adverse impact on product behavior such as dead pressing or desensitization. Video analysis confirmed that displacement was prominent in single hole crater tests but limited in multiple hole tests. Observations confirmed that new fracturing and dilation of discontinuities was evident; with relative differences in fragmentation associated with changes in rock mass conditions [44]. Results from these preliminary trials strongly indicated that the HP explosives are comparable in performance to traditional ammonium nitrate based commercial explosives. 
Waldvogel et al., showed that highly sensitive peroxide based explosives can be dissolved in ionic liquids for a safe handling [45]. Dissolving peroxide explosives such as TATP in lipophilic ionic liquids gives rise to novel and easy to use simulants of as authentic compounds. These solutions are easy to use and may be applied as powerful odor sources for analytical testing and training purposes. Moreover, the stabilizing effect of the ionic liquids can be exploited for a safe sampling of large quantities of these explosives. Such samples of triacetone triperoxide are long-term stable, safe, and valid for forensic evaluations [45]. Solutions of 2\% TATP in 1-methyl-3-octylimidazolium tetrafluoroborate can serve as powerful odor sources, which can simulate large quantities of TATP. Initial studies with HMTD show solubility up to $5 \mathrm{wt}-\%$ and also a strongly decreased sensitivity to friction and impact

\section{Detection}

Most of the current technology in use for trace detection of explosives is unable to detect these energetic compounds. Additionally, differences in physical properties between cyclic organic peroxides make the development of a general method for peroxide analysis and detection difficult. In a book chapter the most relevant contributions related to preparation, characterization and ambient pressure detection of the most important cyclic organic peroxides were reviewed [3]. Characterization analyses are coupled with theoretical calculations and the mechanisms of reaction for TATP, which is the most notorious cyclic organic peroxide [3]. Hexamethylene triperoxide diamine (HMTD) has been the focus of theoretical interest as well. Wierzbicki et al., investigated p- and m-hexamethylene triperoxide diamine systems using density functional theory [46]. They carried out calculations at the level of B3LYP/6-31+G(d). They studied hexamethylene triperoxide diamine (HMTD) in order to elucidate the unusual, nearly planar, $\mathrm{sp}^{2}$ hybridization of the two bridgehead nitrogen atoms, each bonded to the three $\mathrm{CH}_{2}$ groups. They postulated that extended bonding orbitals between the peroxide oxygens results in charge delocalization which decreases lone-pair repulsion and compensates the energy loss due to the $\mathrm{sp}^{3}$ to $\mathrm{sp}^{2}$ hybridization change on the nitrogen atoms. They reexamined the crystal structure of HMTD by performing low-temperature, single-crystal X-ray studies and determined the unit cell contains a 50-50 racemic mixture of enantiomeric forms of HMTD, showing disorder about the mirror plane. At the low temperature, all hydrogen atoms were located and resolved, which was not previously possible. They also crystallized and performed low- 
temperature X-ray analysis of a never previously reported dialdehyde form of HMTD, tetramethylene diperoxide diamine dialdehyde (TMDDD) [46].

In one of the earliest reports of TATP detection in a criminal case study, both GC/MS electron ionization (EI) and chemicals ionization (CI) techniques were used [47]. Since that time the number of GC/MS applications for TATP and HMTD have grown exponentially, today it is one of the prominent techniques for their detection.

In the last few years, the need to determine peroxide-based explosives in solid and air samples has resulted in the development of a series of new analytical methods for triacetonetriperoxide (TATP) and hexamethylenetriperoxidediamine (HMTD). These new analytical methods are critically discussed in the article of Karst et al., [48]. Particular emphasis is placed on spectroscopic and mass spectrometric methods as well as on chromatographic techniques with selective detection schemes. The potential of these methods to analyze unknown solid samples that might contain one or more of the explosives and to analyze peroxide-based explosives in air has been evaluated [48].

In an article by Karst et al. [49], the first method for quantitative trace analysis of peroxide-based explosives is described. In the study, a reversed-phase high-performance liquid chromatography method with post column UV irradiation and fluorescence detection for the analysis of triacetone triperoxide (TATP) and hexamethylene triperoxide diamine (HMTD) was developed. After separation, the analytes were degraded photochemically to hydrogen peroxide, which was subsequently determined on the basis of the peroxidase-catalyzed oxidation of p-hydroxyphenylacetic acid to the fluorescent dimer. This two-step reaction scheme in combination with the respective blanks (photochemical reactor switched off) provides for high selectivity. The limits of detection were obtained as $2 \times 10^{-6} \mathrm{~mol} / \mathrm{L}$ for both TATP and HMTD, respectively. The method has been applied to the analysis of real samples [49].

The detection of TATP and analogues is limited to techniques such as Raman, gas chromatography, mass spectrometry, liquid chromatography and others. UV absorption based methods are not candidates for detection because the molecules lack chromospheres in its structure [50-52]. Many researchers however, have performed studies for specific methods of detection. Buttigieg presented ion-mobility spectrometry (IMS) as a solution for the detection of TATP and HMTD [53]. Others, like Sally and coworkers, have developed methods using HPLC detection [54]. Stambouli et al., used GC/MS techniques for detection of TATP traces in post-explosion debris [55]. Recently, 
highly energetic cyclic organic peroxides detected and analyzed by GC/FT-IR, GC-MS, Raman Microscopy and FT-IR. Also HMTD was analyzed by its decomposition products using MS [50]. These studies confirm the use of GC/MS for trace detection and separation of peroxide from mixtures [50]. A simple and informative method for the characterization of cyclic organic peroxide crystals is high magnification white light microscopy.

The analysis of decomposition products is a modern method of detection for high explosives (HE) [56,57]. Most characterization techniques use methodologies based on gas chromatography (GC) and mass spectrometry (MS). Compounds such as TNT and RDX have been analyzed through this method using GC-MS [58-70]. Most organic peroxide properties allow detection by GC analysis and ion mobility spectrometry (IMS) such as triacetone triperoxide (TATP) and benzoyl peroxide (BPO) [56,57,71-73]. However, other peroxides, such as HMTD, are difficult to analyze through chromatographic methods due to their low vapor pressure and limited solubility [74-77].

Peroxide explosives are difficult to detect by direct optical methods due to their lack of chromophores [78]. Note that both of TATP and DADP are non-fluorescent molecules with no absorption in the UV-VIS. Because of that, a variety of instrumental analytical methods have been developed for their detection, such as electrochemical methods [79], chromatographic and spectroscopic analysis [80,81], mass spectrometry [82] or immunoassays $[83,84]$. However, these detection strategies usually require expensive and non-portable instrumentation and complex sample pretreatment. All these drawbacks can be overcome by using optical chemosensors and probes. However, development of those is much less advanced and most of them are based on the detection of the acid decomposition products of the peroxide explosives, such as $\mathrm{H}_{2} \mathrm{O}_{2}$ or acetone [85,86]. In this sense, it is worth mentioning the colorimetric sensor arrays developed by Suslick's group [87-89]. They have reported a simple and highly sensitive colorimetric sensor for the detection of TATP vapor with semiquantitative analysis from $50 \mathrm{ppb}$ to $10 \mathrm{ppm}$ [87]. By using a solid acid catalyst to pretreat a gas stream, they have discovered that a colorimetric sensor array of redox sensitive dyes can detect even very low levels of TATP vapor from its acid decomposition products (e.g., $\mathrm{H}_{2} \mathrm{O}_{2}$ ) with limits of detection (LOD) below $2 \mathrm{ppb}$. Note that TATP vapors undergo acid-catalyzed decomposition that release detectable volatiles while the much less volatile HMTD contains detectable volatile basic impurities [88]. They have created a simple, disposable colorimetric sensor 
array that is capable of sensitive and semiquantitative detection of the vapor phase of the primary explosive TATP with limits of detection below $0.02 \%$ of its saturation vapor pressure. The array is highly selective for TATP, is unaffected by changes in humidity or by the presence of many common potential interferents, and can differentiate TATP from other chemical oxidants.

Although, peroxide-based explosives, triacetone triperoxide (TATP) and hexamethylene triperoxide diamine (HMTD), can be manufactured from readily accessible reagents, however it is difficult to detect them by conventional analytical methods. To develop a new method of detection, Apak et al., securely synthesized TATP and HMTD, then took up with acetone, and hydrolyzed with $4 \mathrm{M} \mathrm{HCI}$ to hydrogen peroxide [90]. The acidic solution containing $\mathrm{H}_{2} \mathrm{O}_{2}$ was neutralized, and assayed by the spectrophotometric method. The chromophore of the reaction was the chelate absorbing light at $454 \mathrm{~nm}$. The molar absorptivity of the method for TATP and HMTD was $3.45 \mathrm{x}$ $10^{4}$ and $4.68 \times 10^{4} \mathrm{Lmol}^{-1} \mathrm{~cm}^{-1}$, respectively. The TATP recovery from a synthetically contaminated loamy clay soil was $91-99 \%$. The colorimetric method was also applied to a polymeric Nafion membrane sensor developed for the first time in this work for peroxide explosive assay. The absorbance-concentration response was perfectly linear, the limit of detection (LOD) of the procedure for both TATP and HMTD was approximately $0.2 \mathrm{mg} / \mathrm{L}$. Neither common soil ions $\left(\mathrm{Ca}^{2+}, \mathrm{Cl}^{-}, \mathrm{K}^{+}, \mathrm{SO}^{=}, \mathrm{Mg}^{+2}\right.$ and $\mathrm{NO}_{3}^{-}$ at 100-fold amounts nor military-purpose nitro-explosives of TNT, RDX, and PETN at 10-fold amounts interfered with the proposed assay. Active oxygen constituents of laundry detergents (perborates and percarbonates), which normally interfered with the assay, could easily be separated from the analytes by solubility differences. The method was statistically validated against standard reference methods of $\mathrm{TiOSO}_{4}$ colorimetry and GC-MS.

Since existing instrumental methods of analysis are essentially inefficient for the rapid and selective on-site analysis of peroxide based explosives (especially HMTD has a low vapor pressure) which are easily manufactured from readily available reagents, a spectrophotometric procedure coupled to a molecular spectroscopic sensor was devised by the same group. This method was validated against colorimetric and GC-MS standard methods. Once the sample is acid hydrolyzed to hydrogen peroxide, the developed $\mathrm{Cu}$ (II)-neocuproine procedure in neutral medium is very selective and sensitive for TATP and HMTD assay from complex matrices. The method has high selectivity over 
possible interferents existing in nitro-explosive residues. household detergents. reducing sugars. and soil extracts. This is the first colorimetric sensor (naturally excluding microcantilever sensors) ever developed for peroxide explosives. The sensor is of lowcost, is easily applicable to the on-site of determination traces of peroxide-based explosives, and is potentially useful for the analysis of post-blast debris in quick decision making of criminological laboratories

Owing to the lack of UV absorbance, triacetone triperoxide (TATP) is extremely difficult to detect. Apak et al., developed a method which is based on the acidic hydrolysis of TATP into $\mathrm{H}_{2} \mathrm{O}_{2}, \mathrm{pH}$ adjustment to 3.6, and the addition of magnetite nanoparticles $\left(\mathrm{Fe}_{3} \mathrm{O}_{4} \mathrm{MNPs}\right)$ to the medium to produce hydroxyl radicals from $\mathrm{H}_{2} \mathrm{O}_{2}$, owing to the peroxidase-like activity of MNPs [91]. The formed radicals converted the $\mathrm{N}$, Ndimethyl-p-phenylenediamine (DMPD) probe to the colored $\mathrm{DMPD}^{+}$radical cation, the optical absorbance of which was measured at a wavelength of $554 \mathrm{~nm}$. The molar absorptivity $(\varepsilon)$ of the method for TATP was $21.06 \times 103 \mathrm{~L} \mathrm{~mol}^{-1} \mathrm{~cm}^{-1}$. The colored $\mathrm{DMPD}^{+}$product in solution could be completely retained on a cation-exchanger Nafion membrane, constituting a colorimetric sensor for TATP and increasing the analytical sensitivity. They also reported that the proposed method did not respond to a number of hand luggage items like detergent, sweetener, sugar, acetylsalicylic acid (aspirin), and paracetamol-caffeine-based analgesic drugs. On the other hand, TATP could be almost quantitatively recovered from a household detergent and sweetener that can be used as camouflage for the analyte. Neither common soil and groundwater ions (e.g., $\mathrm{Ca}^{2+}, \mathrm{Mg}^{2+}$, $\mathrm{K}^{+}, \mathrm{Cl}^{-}, \mathrm{SO}_{4}{ }^{2-}$, and $\mathrm{NO}_{3}{ }^{-}$) at 100 - fold ratios nor nitro-explosives of trinitrotoluene (TNT), hexahydro-1,3,5-trinitro-1,3,5-triazine (RDX), and pentaerythritol tetranitrate (PETN) at 10-fold amounts interfered with the proposed assay. The method was statistically validated against the standard GC/MS reference method [91].

There are a great many complex techniques for TATP analysis in the literature, but methods for field analysis are limited. Some of these methods are patented, and some require high cost and easily inhibited peroxidase enzymes. The method has high selectivity over possible interfering agents existing in nitro-explosive residues.

In one of their works Oxley and coworkers considered quantification of organic peroxide explosives based on mass spectral analysis [92]. While for analytical purposes, fragments which are not fully characterized in terms of origin and intensity as a function of experimental conditions have been used, understanding the nature of those species is 
very important. In the article they discuss such issues relative to triacetone triperoxide (TATP) and its frequently observed fragment at $\mathrm{m} / \mathrm{z}$ 89. This "fragment" has been identified as the gas-phase reaction product of TATP with one or two methanol molecules/ions. Additionally, the origin and conditions of other fragments at $\mathrm{m} / \mathrm{z} 91,75$, and 74 associated with TATP are addressed. Similar analytical issues associated with other multi-peroxide organic compounds [hexamethylene triperoxide diamine (HMTD) and methyl ethyl ketone peroxides (MEKP)] are discussed. Solution storage conditions for TATP, HMTD, and tetramethylene diperoxide diamine dialdehyde have been determined. While low levels of TATP may be quantified by LC/MS using the molecular fragment $\mathrm{m} / \mathrm{z} 89$, it must be recognized that this fragment has conditions. It represents two different species that are both dependent on the $\mathrm{MeOH}$ concentration, mobile phase modifiers, gas flow, temperature and flow rate. Since the two most common LC/MS solvents for reverse phase chromatography are methanol and acetonitrile, the analyst is faced with a dilemma. If acetonitrile is used as the mobile phase, ionization is suppressed below reasonable levels of analytical detection [93]. If methanol is used, the compound reacts with the species in the gas phase. The gas phase alcohol attack of peroxides (TATP, DADP, MEKP, and HMTD) at the $\alpha$-carbon is apparently a general phenomenon $[94,95]$. This phenomenon can be exploited to lower the limits of detection for these compounds. However, understanding the origins of a particular fragment is very important. The authors remark that all variables must be considered prior to using these ions for quantification. Proper separation must be achieved to prevent unwanted materials (many small compounds may have a mass associated with $\mathrm{C} 4 \mathrm{H}_{9} \mathrm{O}_{2}{ }^{+}$) from providing a false positive response. Also, analytical conditions may significantly affect this particular signal response, making it more susceptible to interference from unknown co-eluting ion suppressors or enhancers. Lastly, other factors may influence the formation of peroxide products [96].

Results have indicated that even a narrow operating range of humidity may produce enough variation (e.g., changing the base peak) to hinder reproducibility for quantitation or mass spectra library matching. Significant changes in molecular adduct ion signal abundance especially impair trace detection and quantitation or MS/MS ion statistics. One remedy is to indirectly control humidity by elevating discharge temperature so that humidity effects are diminished without also causing excessive fragmentation [96].

TATP and DADP having no chromophores are non-fluorescent molecules with no absorption in the UV-vis. Thus, a variety of instrumental analytical methods have been 
developed for their detection, such as electrochemical methods [79,97], chromatographic and spectroscopic analysis $[80,81,98,99]$, mass spectrometry [82,100] or immunoassays $[84,101,102]$. However, these detection techniques/methods usually require expensive and non-portable instrumentation and complex sample pretreatment. However, these drawbacks can be overcome using optical chemosensors and probes. Most of them are based on the detection of the acid decomposition products of the peroxide explosives, such as $\mathrm{H}_{2} \mathrm{O}_{2}$ or acetone $[85,86,103,104]$. In this sense, Suslick's group is to be mentioned by their contribution to the colorimetric sensor arrays development by [8789,105-107].

In a recent article, two dansyl-modified $\beta$-cyclodextrin derivatives have been synthesized as host-guest sensory systems for the direct fluorescent detection of the peroxide explosives diacetone diperoxide (DADP) and triacetone triperoxide (TATP) in aqueous media $[78,108]$. The authors wanted to explore a new approach towards the direct fluorogenic detection of peroxide explosives such as TATP or DADP using hostguest sensory systems [78]. Thus, as suitable hosts they decided to use cyclodextrins (CDs). CDs are cyclic oligosaccharides consisting of $\alpha-1,4-$ linked glucopyranose units. The most common CDs are $\alpha-, \beta$ - and $\gamma$-CDs. TATP and DADP are small hydrophobic molecules, with sizes similar to those of the corresponding branched cycloalkanes. Cyclodextrins are known to form inclusion complexes in aqueous solutions with a large variety of hydrophobic organic molecules of appropriate sizes. The use of chromophoremodified CDs as fluorescent sensors for organic molecules has been extensively developed. Dansyl is the most studied chromophore. The sensing is based on the displacement of the dansyl moiety from the cavity of the cyclodextrin by the peroxide guest resulting in a decrease of the intensity of the fluorescence of the dye. Both systems showed similar fluorescent responses and were more sensitive towards TATP than DADP.

In summary, they have synthesized two new dansyl-appended $\beta$-cyclodextrin sensors and have explored the possibility of using the well-known host-guest chemistry of cyclodextrins for the direct fluorescent sensing of the peroxide explosives DADP and TATP through a displacement approach. The host-guest experiments with $\beta$-cyclodextrin by $1 \mathrm{H}-\mathrm{NMR}$ suggested a good binding of the DADP inside the cavity of the cyclodextrin. Although the preliminary results with sensors developed in aqueous media show a moderate sensitivity, most probably due to solubility problems, this approach 
describes a new strategy for detecting peroxide explosives that has not been previously explored. Both systems showed similar fluorescent responses and both where more sensitive towards TATP than DADP [78].

Another detection method for TATP is based on the precursors of TATP. Thus, in order to detect the precursors of TATP effectively, Sun et al., synthesized molybdenum disulfide/reduced graphene oxide $\left(\mathrm{MoS}_{2} / \mathrm{RGO}\right)$ composites by a hydrothermal method, using two-dimensional reduced graphene oxide (RGO) as template [109]. Graphene materials have been widely explored for the fabrication of gas sensors because of their atom thick two-dimensional conjugated structures, high conductivity and large specific surface area. The effects of the ratio of RGO to raw materials for the synthesis of $\mathrm{MoS}_{2}$ on the morphology, structure, and gas sensing properties of the $\mathrm{MoS}_{2} / \mathrm{RGO}$ composites were studied. It was found that after optimization, the response to $50 \mathrm{ppm}$ of $\mathrm{H}_{2} \mathrm{O}_{2}$ vapor was increased from $29.0 \%$ to $373.1 \%$, achieving an increase of about 12 times. Meanwhile, all three sensors based on $\mathrm{MoS}_{2} / \mathrm{RGO}$ composites exhibited excellent antiinterference performance to ozone with strong oxidation. Furthermore, three sensors based on $\mathrm{MoS}_{2} / \mathrm{RGO}$ composites were fabricated into a simple sensor array, realizing discriminative detection of three target analytes in $14.5 \mathrm{~s}$ at room temperature. This work shows that the synergistic effect between two-dimensional RGO and $\mathrm{MoS}_{2}$ provides new possibilities for the development of high performance sensors [109].

In recent years, many articles on the detection of $\mathrm{H}_{2} \mathrm{O}_{2}$ have been reported. Their applications include environmental, biological, food, and industrial fields, using such as the non-enzymatic chemi-resistive $\mathrm{H}_{2} \mathrm{O}_{2}$ sensor [110,111]. The synergistic effect between two-dimensional RGO and $\mathrm{MoS}_{2}$ provide new possibilities for the development of high performance sensors, [112-114], which achieve the advantages of low cost detection. In fact, simultaneous detection of $\mathrm{H}_{2} \mathrm{O}_{2}$ and $\mathrm{C}_{3} \mathrm{H}_{6} \mathrm{O}$ would provide a viable basis for the detection of explosive TATP. Of course using electrochemical detection of TATP precursors is not a new concept [87,115-117]. Dobrokhotov's [118] team introduced a good response with nano-spring-based sensors for gases such as TATP precursors, as well as determining conditions for $\mathrm{In}_{2} \mathrm{O}_{3}$ [119] and $\mathrm{WO}_{3}$ [120] but still did not achieve operation at room temperature. The application of graphene in chemical gas sensors is receiving more and more attention [121,122].

p-Type RGO and n-type $\mathrm{MoS}_{2}$, typical two-dimensional nanomaterials, were used 
successfully to design hierarchical $\mathrm{MoS}_{2} / \mathrm{RGO}$ composites using RGO as templates [109]. The effects of the ratio of RGO to raw materials for the synthesis of $\mathrm{MoS}_{2}$ on the morphology, structure, and gas sensing properties of the $\mathrm{MoS}_{2} / \mathrm{RGO}$ composites were studied in order to detect the precursors of TATP effectively. It was found that after optimization, the response to $50 \mathrm{ppm}$ of $\mathrm{H}_{2} \mathrm{O}_{2}$ vapor was increased from $29.0 \%$ to $373.1 \%$, achieving an increase of about 12 times. Meanwhile, all three sensors based on $\mathrm{MoS}_{2} / \mathrm{RGO}$ composites exhibited excellent anti-interference performance to ozone with strong oxidation. Furthermore, the simple sensor array based on $\mathrm{MoS}_{2} / \mathrm{RGO}$ composites achieved discriminative detection of three target analytes in $14.5 \mathrm{~s}$ at room temperature. This proves that the design of gas sensing properties of $\mathrm{MoS}_{2} / \mathrm{RGO}$ composites by changing the ratio of RGO to $\mathrm{MoS}_{2}$ is effective and feasible. The synergistic effect between two-dimensional RGO and $\mathrm{MoS}_{2}$ provide new possibilities for the development of high performance sensors. There exist recent advancements on the synthesis of graphene materials for this purpose and the techniques applied for fabricating gas sensors. The effects of the compositions, structural defects and morphologies of graphene-based sensing layers and the configurations of sensing devices on the performances of gas sensors have been discussed by Shi and Yuan [122].

Burks and Hage have reviewed current trends in the detection of peroxide-based explosives with a focus on luminescence and fluorescence methods, infrared and Raman spectroscopy, mass spectrometry, and electrochemical techniques [123]. Also some newer developments in gas chromatography and high performance liquid chromatography methods are discussed. One recent trend that is discussed is an emphasis on field measurements through the use of portable instruments or portable assay formats. An increase in the use of infrared spectroscopy and mass spectrometry for peroxide based explosives (PBE) analysis is also noted. The analysis of triacetone triperoxide has been the focus in the development of many of these methods, although hexamethylene triperoxide diamine has received increased attention in PBE detection during the last few years.

A simple and selective colorimetric method for the detection of perilous peroxide explosives has been developed by Bagheri et al., [124] based on the peroxidase mimetic activity of silver nanoparticles/flake-like zinc metal-organic framework nanocomposite (Ag@ZnMOF). The synthesis of Ag@ZnMOF has been achieved. The composite contained the formation of silver nanoparticles (AgNPs) inside the fine pores of $\mathrm{Zn}$ 
metal-organic framework ( $\mathrm{ZnMOF})$. High reactive $\mathrm{AgNPs}$ as well as great surface area of MOFs provided a synergetic and high improved catalytic activity for the composite which was studied as a peroxidase mimic in hydrogen peroxide $\left(\mathrm{H}_{2} \mathrm{O}_{2}\right)$-based oxidations. The achieved system was used for detection of triacetone triperoxide (TATP) as one of the most hazardous peroxide explosives. In fact, TATP was decomposed in an acidic condition to generate $\mathrm{H}_{2} \mathrm{O}_{2}$, which was then applied to oxidize 3,3',5,5'tetramethylbenzidine (TMB) in the presence of the Ag@ZnMOF as a catalyst. This reaction produces the well-known blue colored charge transfer complex of the dye (oxTMB), which was recognized by the colorimetric technique. A linear relationship was obtained between the absorption intensity of the produced blue solution and the TATP concentration in the range of $0.4-15 \mathrm{mg} \mathrm{L}^{-1}$, with a detection limit of $0.1 \mathrm{mg} \mathrm{L}^{-1}$. Also a portable test kit was prepared using the same reagents for TATP measurement in real samples [124].

In the study of Zhang and coworkers, chromium oxide $\left(\mathrm{Cr}_{2} \mathrm{O}_{3}\right)$ nanoparticles were found to greatly enhance the chemiluminescence (CL) of the luminol-hydrogen peroxide $\left(\mathrm{H}_{2} \mathrm{O}_{2}\right)$ system [125]. A novel microarray $\mathrm{CL}$ method was originally developed for the detection of triacetone triperoxide (TATP). The novel CL method was based on the outstanding catalytic effect of a $\mathrm{Cr}_{2} \mathrm{O}_{3}$ nanoparticle array on the CL reaction between lower concentrations of luminol and $\mathrm{H}_{2} \mathrm{O}_{2}$, which come from hydrolysis of TATP vapor. Generally, the mechanism of the luminol- $\mathrm{H}_{2} \mathrm{O}_{2}$ CL reaction is related to the reaction between luminol radical, superoxide radical, and hydroxyl radical. The surface of metal oxide nanoparticles can be oxidized to form $\mathrm{O}-\mathrm{O}$ bond which can cause decomposition to yield active radical intermediates such as $\mathrm{OH}$ and $\mathrm{O}_{2}$. The calibration curve of $\mathrm{H}_{2} \mathrm{O}_{2}$ was found to be linear over a range of $1.0 \times 10^{-8}$ to $3.0 \times 10^{-5} \mathrm{M}$ with a detection limit of $1.6 \times 10^{-9} \mathrm{M}\left(R^{2}=0.9992, n=12\right)$. The $\mathrm{CL}$ method has the advantages of being sensitive, selective, simple, time-saving and shows good reproducibility. Therefore, this technique can also be used in a variety of applications, such as environmental monitoring, industrial and food processing, biomedical technology, healthcare, and clinical analysis, thus all these would make it easily popular.

The use of improvised explosive devices (IEDs) threatens public at an alarming rate. However, many devices for IEDs detection generally lacks sensitivity, reliability, portability. In the work of Mbah and coworkers, they fabricated a disposable solid phase electrolyte/electrode (SPEE) interfaces for the detection of trace quantities of peroxide 
based explosives, namely, triacetone triperoxide (TATP) and hexamethylene triperoxide diamine (HMTD) which were synthesized without recrystallization, from their precursors: $\mathrm{H}_{2} \mathrm{O}_{2}$ and acetone via electrochemical technique [126]. The SPEE device was able to detect $8.66 \pm 1.048 \mathrm{ng}$ of TATP at its vapor pressure of $4.80 \mathrm{~Pa}$ at $298 \mathrm{~K}$. However, the usability of SPEE is restricted to first time use only due to the failure encountered while attempting to reuse it, and needs to be replaced. As suggested by the electrochemical cell impedance test, mass transfer limitations caused by the solution and charge transfer resistances at the electrode interface impedes reaction kinetics. Nevertheless, the ease of fabrication, response and speed make SPEE sensor an excellent choice and offer opportunity for the development of miniature sensors for screening of IEDs and its precursors at various screening sites.

Furthermore, the sensing of the precursors in mixtures of other materials (as demonstrated by acetone in strengthening nail polish remover) or at different concentrations as shown by $\mathrm{H}_{2} \mathrm{O}_{2}$ has been carried out successfully. Further analysis conducted to investigate the electrochemical cell impedance behavior has indicated that mass transfer limitations, slow electrode kinetics and covering of the electrode surface by reaction products all might have contributed to the observed failure after the first time usage. Since, many sensors cannot detect the required low levels of explosive vapors, the SPEE sensor offer an opportunity that can be exploited for development of miniature sensor devices for screening of IEDs and their precursors [126].

Biosensors for the detection of peroxide explosives also exist in the literature. For the development of biosensors, immunochemical $\mu$-TAS, electronic noses, immunological test kits, or test strips, the availability of antibodies of high quality is crucial [84]. Quite recently, Weller and coworkers presented the successful immunization of mice, based on the design, synthesis, and conjugation of a novel TATP derivative [84]. In the study, the long-term immunization of rabbits has been shown, which resulted in antibodies of extreme selectivity and more than 1,000 times better affinity in relation to the antibodies from mice. Detection limits below $10 \mathrm{ng} \mathrm{L}^{-1}$ (water) were achieved. The cross-reactivity tests revealed an extraordinary selectivity of the antibodies-not a single compound could be identified as a relevant cross-reactant. The presented immunoreagent might be a major step for the development of highly sensitive and selective TATP detectors particularly for security applications [84]. 


\section{Hazardous Effects and Metabolism}

Many oxygenated organic compounds become increasingly dangerous upon prolonged storage because they tend to form explosive peroxides with age. Peroxides may form in freshly distilled, undistilled, and unstabilized ethers within less than two weeks. Exposure to light and air enhances the formation of the peroxides. Many ethers tend to absorb and react with oxygen from the air to form unstable peroxides which may detonate with extreme violence when they become concentrated by evaporation or distillation. These ethers may also explode when combined with other compounds that produce a detonable mixture or when disturbed by heat, shock, or friction. Peroxides formed in compounds by autooxidation have caused many accidents, including the unexpected explosions of the residue of solvents after distillation. While ethers present one of the most commonly known peroxidizable compound risks, other common solvents such as isopropanol have exploded upon distillation due to peroxide concentration. With all peroxide forming chemicals it is preferable to use small containers that can be completely emptied, rather than take small amounts from a large container over time. Ethers should be stored in amber bottles or other opaque containers and under a blanket of inert gas, such as nitrogen or argon, or over a reducing agent to inhibit formation of peroxides. Containers of ether and other peroxide forming chemicals should be marked with date received and date opened [127].

Oxley et al., interested metabolism of some peroxide explosives [128]. Triacetone triperoxide (TATP) and hexamethylene triperoxide diamine (HMTD) are prominent peroxide explosives. Mitigation of peroxide explosives is a priority among the law enforcement community, with canine (K9) units being trained to recognize the scent of peroxide explosives. In their study, the metabolism, blood distribution, and toxicity of peroxide explosives are investigated. Metabolism studies on HMTD two potential metabolites in liver microsomes identified, as tetramethylene diperoxide diamine alcohol aldehyde (TMDDAA) and tetramethylene peroxide diamine dialcohol dialdehyde (TMPDDD). Blood stability studies revealed that in dogs and humans HMTD was rapidly degraded, whereas TATP remained for at least one week. Toxicity studies in dog and human hepatocytes indicated minimum cell death for both TATP and HMTD.

Oxley et al., performed some in vitro experiments using human liver microsomes and recombinant enzymes on TATP and TATP-OH for metabolite identification and enzyme phenotyping [129]. Enzyme kinetics for TATP hydroxylation were also investigated. 
Urine samples were collected from laboratory personnel before and after working with TATP. They were analyzed for TATP and its metabolites. While experiments with flavin monooxygenases were inconclusive, those with recombinant cytochrome P450s (CYPs) strongly suggested that CYP2B6 was the principle enzyme responsible for TATP hydroxylation. Also, TATP-O-glucuronide was identified and incubations with recombinant uridine diphosphoglucuronosyltransferases (UGTs) indicated that UGT2B7 catalyzes this reaction. Kinetic studies were performed. Michaelis-Menten kinetics were determined for TATP hydroxylation, which yielded $K_{\mathrm{m}}=1.4 \mu \mathrm{M}$ and $V_{\max }=8.7$ $\mathrm{nmol} / \mathrm{min} / \mathrm{nmol}$ CYP2B6. TATP-O-glucuronide was present in the urine of all three volunteers after being exposed to TATP vapors. The results showed good accordance between in vivo and in vitro data. TATP and TATP-OH were not observed.

Since scientists working to characterize and detect TATP to prevent terrorist attacks are constantly exposed to this volatile compound, attention should be paid to its metabolism. This paper is the first to elucidate some exposure, metabolism and excretion of TATP in humans and to identify a marker of TATP exposure, TATP-O-glucuronide in urine.

The toxicity or pharmacodynamics of many of the nitrated explosives have been well documented. Peroxide explosives have been on the rise due to the ease of manufacturing from household products. Of particular interest are the cyclic peroxides triacetone triperoxide (TATP) and hexamethylene diamine triperoxide (HMTD). Very little is known about the toxicity or potentially beneficial effects of these compounds. This may be primarily due to the difficulty in detecting or working with these materials, particularly when they are extracted from living tissues. The use of liquid chromatography (LC)/mass spectrometry (MS) is ideally suitable to handle this type of sample, provided that the proper detection limits can be achieved. Additionally, this technique provides a very sensitive detection with gentle ionization for more definitive confirmation of the chemical in question over many other techniques historically chosen.

In the study of Kolizza, several remarkable discoveries were made while trying to reduce the limits of quantification for TATP and HMTD [130]. For example, acetonitrile, one of the most commonly used LC/MS solvents has been observed inhibiting ionization directly. The author proposed a mechanism for this suppression that is by the formation of neutral aggregates of the nitrile moiety with various, common functional groups. Peroxides are one of the most intensely affected moieties. Also, TATP and methyl ethyl 
ketone peroxide (MEKP) have been shown to react with one or more alcohols under atmospheric pressure ionization (API) conditions to produce new species which may be exploited to improve limits of detection. One has to be cautious while working with these products since the conditions can directly affect the signal intensity and multiple related analytes can all provide this common product. Lastly, HMTD has been found to react with both primary and secondary amines and alcohols in the gas phase to produce unique products related to the nature of the amine or alcohol. This research has allowed limits of detection to improve by 20 to 50 times our original analysis limits.

Toxicity of HMTD and TATP was the prime question in the study. Simple in vitro analysis using canine liver microsomes (DLM) and lung microsomes (DLgM) in the presence of NADPH (electron donor) were performed to determine the rate, product and nature of the metabolism [130]. The results have indicated that the metabolism that does occur appears to be NADPH-dependent, which limits the types of enzymes with may be responsible. The affinity for the non-specific metabolism is very high, with a $\mathrm{K}_{\mathrm{m}}$ value of $2.21 \mu \mathrm{M}( \pm 14.8 \%)$ with a $\mathrm{V}_{\max }$ of $1.13 \mathrm{nmol} / \mathrm{min} / \mathrm{mg}$ protein $( \pm 3.27 \%)$. This also indicates that the enzyme responsible for the metabolism is saturated at relatively low concentrations. Work with recombinant isoforms of specific CYP enzymes (rCYP) has shown that only rCYP2B11 has any effect and that this metabolism is enhanced by the presence of cytochrome b5. The metabolism of CYP2B11 does not seem to account for all of the total metabolism of TATP. Only one metabolite has been identified, the mono-oxidation of a single primary methyl carbon (TATP-OH), for TATP [130].

Oxley et al., in one of their works tried to determine whether there is a potential for the accumulation and toxicity of triacetone triperoxide (TATP) in canines [131]. Additionally this information may suggest human toxicity of TATP and could give some clues about possible detection of biomarkers, metabolites or intact molecule of those using this material for nefarious reasons.

Liquid chromatography/mass spectrometry was used to measure substrate depletion of dog liver microsome (DLM) incubation samples of TATP. Trapping of electrophilic products was performed using glutathione (GSH) and semicarbazide. Then, comparisons were made to free hydroperoxides found in methyl ethyl ketone peroxides (MEKP).

The non-specific $K_{\mathrm{m}}$ value of $2.2 \mu \mathrm{M}$ and a $V_{\max }$ of $1.1 \mathrm{nmol} / \mathrm{min} / \mathrm{mg}$ of protein were determined. Canine recombinant cytochrome P450 (rCYP) $2 \mathrm{~B} 11$ with human cytochrome b5 was found to catalyze the NADPH-dependent metabolism of TATP into 
its only phase I metabolite, hydroxy-TATP (TATP-OH). No secondary metabolite(s) or degraded products were detected or trapped from microsomal incubations. On the other hand, MEKP subjected to similar conditions was found to undergo significant metabolism, semicarbazide trapping and rapid oxidation of GSH to disulfide, GSSG. The synthesized TATP-OH metabolite incubated in DLM progressed three times faster than TATP metabolism with no secondary metabolites found or trapped.

TATP was observed not reacting as MEKP suggesting that TATP does not ring-open to form hydroperoxides. TATP and TATP-OH compete for the same enzyme, with TATP dominating this competition. Failure to detect additional metabolite(s) suggests they may be too small to detect by the system employed or bound covalently to a protein or polymer in the incubation reaction [131].

\section{Conclusion}

The present short review, outlines the most relevant contributions related to preparation, characterization and detection of the most important cyclic organic peroxides. It also includes few recent investigations about the toxicity and metabolism of some peroxide explosives. Although in recent years a variety of instrumental analytical methods have been developed for the detection of peroxide explosives, such as electrochemical methods, chromatographic and spectroscopic analysis, mass spectrometry or immunoassays, the research about the detection of them has been concentrated on more practical techniques using optical chemosensors and probes. On the other hand, progressively increasing effort has been devoted to understanding of metabolism of those chemicals and establishing detection methods based on their metabolites.

\section{Some frequently used abbreviations}
AN : Ammonium nitrate
CDs : Cyclodextrins
CL : Chemiluminescence
DADP : Diacetone di peroxide
DLM : Canine liver microsomes
EDD : Explosive detection dog 
HMEs : Homemade explosives

HMTD : Hexamethylene triperoxide diamine

HP : Hydrogen peroxide

HPOM : Hydrogen peroxide-organic matter

IEDs : Improvised explosive devices

MEKP : Methyl ethyl ketone peroxide

OPEs : Organic peroxide explosives

PBE : Peroxide based explosives

RGO : Reduced graphene oxide

SPEE : Solid phase electrolyte/electrode

TATP : Triacetone triperoxide

TMDD : Tetramethylene diperoxide dicarbamide

TPTP : Tripentanone triperoxide

\section{References}

[1] T.M. Klapötke, Chemistry of High Energy Materials, Berlin: De Gruyter, 2011. https://doi.org/10.1515/9783110439335

[2] R. Matyas and J. Pachman, Primary Explosives, Berlin: Springer-Verlag, 2013. https://doi.org/10.1007/978-3-642-28436-6

[3] E.A. Espinosa-Fuentes, A.J. Peña-Quevedo, L.C. Pacheco-Londoño, R. Infante-Castillo and S.P. Hernández-Rivera, A Review of Peroxide Based Homemade Explosives: Characterization and Detection, in: Explosive Materials: Classification, Composition and Properties; Chemical Engineering Methods and Technology Series, (Editor: Janssen T.J.), NY: Nova Science Publishers, Inc., 2010.

[4] G. Rarata and J. Smętek, Explosives based on hydrogen peroxide - A historical review and novel applications, Materiaty Wysokoenergetyczne / High-Energetic Materials 8 (2016), 56-62.

[5] M.S. Bali, Novel strategies for the safe chemical degradation of organic peroxide explosives: A mechanistic investigation, $\mathrm{PhD}$ thesis, School of Physical, Environmental and Mathematical Sciences, The University College, UNSW Canberra, November, 2014. 
[6] N.A. Milas and A. Golubovic, Studies in organic peroxides. XXV. Preparation, separation and identification of peroxides derived from methyl ethyl ketone and hydrogen peroxide, J. Am. Chem. Soc. 81(21) (1959), 5824-5826.

https://doi.org/10.1021/ja01530a068

[7] E. Riedel and C.H. Janiak, Anorganische Chemie, Berlin: Walter de Gruyter, 2007. https://doi.org/10.1515/9783110206869-011

[8] G.P. Kennedy, Vengeance Weapon 2: The V-2 Guided Missile. Washington DC: Smithsonian Institution Press, 1983.

[9] B.J. Ford, Secret Weapons, Oxford: Osprey Publishing, 2011.

[10] J. Christpher, The Race for Hitler's X-Planes, The Mill, Gloucestershire: History Press, 2013.

[11] R. Wolffenstein, Ueber die Einwirkung von Wasserstoffsuperoxyd auf Aceton und Mesityloxyd, Ber. Dtsch. Chem. Ges. 28(2) (1895), 2265-2269.

https://doi.org/10.1002/cber.189502802208

[12] R. Matyas and J. Pachman, Study of TATP: Influence of reaction conditions on product composition, Propellants Explos. Pyrotech. 35 (2010), 31-37.

https://doi.org/10.1002/prep.200800044

[13] H. Jiang, G. Chu, H. Gong and Q. Qiao, Tin chloride catalyzed oxidation of acetone with hydrogen peroxide to tetrameric acetone peroxide, J. Chem. Res. 28(4) (1999), 288-289. https://doi.org/10.1039/a809955c

[14] K.B. Landenberger, O. Bolton and A.J. Matzger, Two isostructural explosive cocrystals with significantly different thermodynamic stabilities, Angew. Chem. Int. Ed. 52 (2013), 6468-6471. https://doi.org/10.1002/ange.201302814

[15] K. B. Landenberger, O. Bolton and A. J. Matzger, Energetic-energetic cocrystals of diacetone diperoxide (DADP): dramatic and divergent sensitivity modifications via cocrystallization, J. Am. Chem. Soc. 137 (2015), 5074-5079.

https://doi.org/10.1021/jacs.5b00661

[16] N. Milas, R.S. Harris and A. Golubovic, Detection separation and identification of organic peroxides, Radiation Research Supplement, Vol. 3, Implications of Organic Peroxides in Radiobiology. Proceedings of an International Symposium Sponsored by Division of Biological and Medical Research, Argonne National Laboratory, Argonne, Illinois, May 7- 9, 1962 (1963), pp. 71-92.

[17] B.W. Stiasny, Investigation of Organic Peroxides and Their Properties as Energetic 
Materials. Ph.D. Dissertation, Ludwig Maximilian University of Munich, Munich, Germany, 2016.

[18] V. Bulatov, O. Reany, R. Grinko, I. Schechter and E. Keinan, Time-resolved, laser initiated detonation of TATP supports the previously predicted non-redox mechanism, Phys. Chem. Chem. Phys. 15(2013), 6041-6048. https://doi.org/10.1039/c3cp44662j

[19] Y. Li, H. Hao, Q. Zhang and Y. Wu, A broadly applicable mild method for the synthesis of gem-diperoxides from corresponding ketones or 1,3 dioxolanes, Org. Lett. 7 (2009), 1615-1618. https://doi.org/10.1021/o1900262t

[20] N-D.H. Gamage, B. Stiasny, J. Stierstorfer, P.D. Martin, T.M. Klapötke and C.H. Winter, Highly energetic, low sensitivity aromatic peroxy acids, Chem. Eur. J. 22 (2016), 25822585. https://doi.org/10.1002/chem.201502989

[21] M.R. Miner and K.A. Woerpel, $\mathrm{Cu}^{\mathrm{I}}$-catalyzed synthesis of propargyl hydroperoxides using molecular oxygen and hydroxylamines, Eur. J. Org. Chem. 10(2016), 1860-1866. https://doi.org/10.1002/ejoc. 201600038

[22] Y. Sun, N. Li, X. Xing, X. Zhang, Z. Zhang, G. Wang, J. Cheng and Z. Hao, Catalytic oxidation performances of typical oxygenated volatile organic compounds (acetone and acetaldehyde) over MAlO (M= Mn, Co, Ni, Fe) hydrotalcite-derived oxides, Catalysis Today 327 (2019), 389-397. https://doi.org/10.1016/j.cattod.2018.03.002

[23] T.J. Kim, N.H. Heo, J. Kim and G. Seo, Formation of acetone cyclic triperoxide over titania-incorporated mesoporous materials, React. Kinet. Catal. Lett. 79 (2003), 287-293. https://doi.org/10.1023/A:1024538219186

[24] P. Ghorai and P. Dussault, Mild and efficient Re(VII)-catalyzed synthesis of 1,1dihydroperoxides, Org. Lett. 20 (2008), 4577-4579. https://doi.org/10.1021/ol801859c

[25] A.E-F. Eduardo, C. P-L. Leonardo and A. B-C. Marcos, Novel uncatalyzed synthesis and characterization of diacetone diperoxide, Propellants Explos. Pyrotech. 37(4) (2012), 413-421. https://doi.org/10.1002/prep.201000130

[26] I.A. Yaremenko, V.A. Vil', D.V. Demchuk and A.O. Terent'ev, Rearrangements of organic peroxides and related processes, Beilstein J. Org. Chem. 12 (2016), 1647-1748. https://doi.org/10.3762/bjoc.12.162

[27] M.S. Bali, L.Wallace, A.I. Day and D. Armitt, Cyclic pentanone peroxide: Sensitiveness and suitability as a model for triacetone triperoxide, Journal of Forensic Sciences 59 (2014), 936-942. https://doi.org/10.1111/1556-4029.12439

[28] M.S. Bali, D. Armitt, L. Wallace and A.I. Day, Rapid degradation of cyclic peroxides by 
titanium and antimony chlorides, Dalton Transactions 44 (2015), 6775-6783.

http://dx.doi.org/10.1039/c5dt00200a

[29] R.I. Hiyoshi, J. Nakamura and T.B. Brill, Thermal decomposition of organic peroxides TATP and HMTD by T-Jump/FTIR spectroscopy, Propellants Explos. Pyrotech. 32(2) (2007), 127-134. https://doi.org/10.1002/prep.200700002

[30] V.P. Sinditskii, V.I. Kolesov, V.Yu. Egorshev, D.I. Patrikeev and O.V. Dorofeev, Thermochemistry of cyclic acetone peroxides, Thermochimica Acta 585 (2014), 10-15. https://doi.org/10.1016/j.tca.2014.03.046

[31] J. Oxley, J. L. Smith, J. Huang and W. Luo, Destruction of peroxide explosives, Journal of Forensic Sciences 54(5) (2009),1029-33.

https://doi.org/10.1111/j.1556-4029.2009.01130.x

[32] J. C. Oxley, J. L. Smith, and H. Chen, Decomposition of a multi-peroxidic compound: triacetone triperoxide (TATP), Propellants, Explosives, Pyrotechnics 27 (2002),209-216. https://doi.org/10.1002/1521-4087(200209)27:4<209::AID-PREP209>3.0.CO;2-J

[33] F. Dubnikova, R. Kosloff, J. Almog, Y. Zeiri, R. Boese, H. Itzhaky, A. Alt and E. Keinan, Decomposition of triacetone triperoxide is an entropic explosion, J. Am. Chem. Soc. 127 (2005), 4, 1146-1159. https://doi.org/10.1021/ja0464903

[34] Y.B. Tsaplev, Decomposition of cyclic acetone peroxides in acid media, Kinet. Catal. 53 (2012), 521-524. https://doi.org/10.1134/S0023158412050163

[35] T.M. Klapötke and T. Wloka, Peroxide Explosives, Wiley online library, 2014. https://doi.org/10.1002/9780470682531.pat0879

[36] Home-made Explosives, 2016.

http://www.3dchem.com/moremolecules.asp?ID=312\&othername=TATP

[37] Triacetone Triperoxide (TATP), http://www.globalsecurity.org/military/systems/munitions/tatp.htm

[38] Home-made Explosives, http://www.3dchem.com/molecules.asp?ID=313

[39] Hexamethylene Triperoxide Diamine (HMTD), http://www.3dchem.com/molecules.asp?ID=427

[40] R. Matyás and J. Selesovský, Power of TATP based explosives, J. Hazard. Mater. 165(13) (2009), 95-9. https://doi.org/10.1016/j.jhazmat.2008.09.063.

[41] G.J. McKay, Forensic characteristics of organic peroxide explosives (TATP, DADP, and HMTD), Kayaku Gakkaishi (J. of the Japan Explosives Soc.) 63(6) (2002), 323-329. 
[42] M. Araos and I.A. Onederra, Study of the detonation process of novel hydrogen peroxidebased explosives using high speed video, Forty-Third Annual Conference on Explosives and Blasting Technique, Orlando, Florida USA, 29 Jan-1 Feb 2017, Cleveland, OH USA: International Society of Explosives Engineers.

[43] L.T. R. Dobson, Ultrasonic Activation of Triacetone Triperoxide, University of Nebraska, 2010.

[44] I. Onederra and M. Araos, Preliminary quantification of the in situ performance of a novel hydrogen peroxide based explosive, Mining Technology 126(2) (2017), 113-122. https://doi.org/10.1080/14749009.2017.1290336

[45] D. Lubczyk, A. Hahma, M. Brutschy, C. Siering and S.R. Waldvogel, A new reference material and safe sampling of terrorists peroxide explosives by a non-volatile matrix, Propellants Explos. Pyrotech. 40(4) (2015), 590-594.

https://doi.org/10.1002/prep.201500011

[46] A. Wierzbicki, E.A. Slater, E.A. Cioffi and E.D. Stevens, Density functional theory and $\mathrm{X}$-ray investigations of $\mathrm{P}$ - and $\mathrm{M}$-hexamethylene triperoxide diamine and its dialdehyde derivative, J. Phys. Chem. A 105(2001), 8763-8768. https://doi.org/10.1021/jp0123841

[47] H.K. Evans, F.A.J. Tulleners, B.L. Sanchez and C.A. Rasmussen, An unusual explosive, triacetonetriperoxide (TATP), J. Forensic Sci. 31 (1986), 1119-1125. https://doi.org/10.1520/JFS11122J

[48] R. Schulte-Ladbeck, M. Vogel and U. Karst, Recent methods for the determination of peroxide-based explosives, Anal. Bioanal. Chem. 386 (2006), 559-565. https://doi.org/10.1007/s00216-006-0579-y

[49] R. Schulte-Ladbeck, P. Kolla and U. Karst, Trace analysis of peroxide-based explosives, Anal. Chem. 75(4) (2003), 731-735. https://doi.org/10.1021/ac020392n

[50] D.S. Viswanath, T.K. Ghosh and V.M. Boddu, Triacetone Triperoxide (TATP), in: Emerging Energetic Materials: Synthesis, Physicochemical, and Detonation Properties, Amsterdam: Springer Netherlands, 2018, pp. 273-291.

https://doi.org/10.1007/978-94-024-1201-7

[51] M.S. Meaney and V.L. McGuffin, Luminescence-based methods for sensing and detection of explosives, Anal. Bioanal. Chem. 391 (2008), 2557-2576. https://doi.org/10.1007/s00216-008-2194-6

[52] R. Matyas, J. Pachman and H. Ang, Study of TATP: Spontaneous transformation of TATP to DADP, Propellants Explosives Pyrotechnics 33(2) (2008), 89-91. https://doi.org/10.1002/prep.200700247 
[53] G. Buttigieg, A. Knight, S. Denson, C. Pommier and M. Denton, Characterization of the explosive triacetone triperoxide and detection by ion mobility spectrometry, Forensic Science International 135 (2003), 53-59. https://doi.org/10.1016/S0379-0738(03)00175-0

[54] S.C. Gamble, L.C. Campos and R.M. Morgan, Detection of trace peroxide explosives in environmental samples using solid phase extraction and liquid chromatography mass spectrometry, Environmental Forensics 18(1) (2017), 50-61. https://doi.org/10.1080/15275922.2016.1263901

[55] A. Stambouli, A. El Bouri, T. Bouayoun and M.A. Bellimam, Headspace-GC/MS detection of TATP traces in post-explosion debris, Forensic Science International $146 \mathrm{~S}$ (2004) 191-194. https://doi.org/10.1016/j.forsciint.2004.09.060

[56] J. Yinon, Advances in Forensic Applications of Mass Spectrometry, Boca Raton, FL: CRC Press, 2004, p. 279.

[57] J.C. Oxley, J.L. Smith, H. Chen and E. Cioffi, Decomposition of multi-peroxidic compounds: Part II. Hexamethylene triperoxide diamine (HMTD), Thermochimica Acta 388 (1-2) (2002), 215-225. https://doi.org/10.1016/S0040-6031(02)00028-X

[58] B. Báez, S.N. Correa and S.P. Hernandez-Rivera, Transport of explosives II: use of headspace-SPME/GC $\mu$-ECD and TEEM GC/MS for detection of TNT vapors from sand buried samples, Proc. SPIE Int. Soc. Opt. Eng. 5794 (2005), 1263-1271. https://doi.org/10.1117/12.602446

[59] J. Leppert, M. Härtel, T.M. Klapötke and P. Boeker, Hyperfast flow-field thermal gradient GC/MS of explosives with reduced elution temperatures, Anal. Chem. 90 (14) (2018), 8404-8411. https://doi.org/10.1021/acs.analchem.8b00900

[60] S.N. Correa-Torres, M. De Jesús, N. Mina-Camilde, M.E. Castro, A. Blanco, S.P. Hernandez-Rivera, R.B. Cody and J.A. Laramee, Improved detection of landmine components: using TEEM-GC-MS for detection of TNT and RDX in soil and other complex matrices, Proc. SPIE Int. Soc. Opt. Eng. 5089 (2003), 1001-1011. https://doi.org/10.1117/12.487224

[61] V. Florian, A. Cabanzo, B. Baez, S. Correa, M. Irrazabal, J. G. Briano, M. E. Castro and S.P. Hernandez-Rivera, Detection of the spectroscopic signatures of explosives and their degradation products, Proc. SPIE Int. Soc. Opt. Eng. 5794 (2005), 724-728.

[62] J.C. Oxley, J.L. Smith, L.J. Kirschenbaum, S. Marimganti and S. Vadlamannati, Detection of explosives in hair using ion mobility spectrometry, J Forensic Sci. 53(3) (2008), 690-3. https://doi.org/10.1111/j.1556-4029.2008.00719.x 
[63] A. J. Peña-Quevedo, R. Cody, N. Mina-Camilde, M. Ramos, and S. P. HernandezRivera, Characterization and differentiation of high energy amine peroxides by direct analysis in real time TOF/MS, Proc. SPIE 6538 (2007), 653828-12. https://doi.org/10.1117/12.720577

[64] W.P. Schaefer, J.T. Fourkas and B.G. Tiemann, Structure of hexamethylene triperoxide diamine, J. Am. Chem. Soc. 107(8) (1985), 2461-2463.

https://doi.org/10.1021/ja00294a043

[65] X. Xu, A.M. van de Craats, E.M. Kok and P.C. de Bruyn, Trace analysis of peroxide explosives by high performance liquid chromatography-atmospheric pressure chemical ionization-tandem mass spectrometry (HPLC-APCI-MS/MS) for forensic applications, $J$. Forensic Sci. 49 (6) (2004), 1230-6.

[66] R.B. Cody, J.A. Laramee and H.D. Durst, Versatile new ion source for the analysis of materials in open air under ambient conditions, Anal. Chem. 77 (2005), 2297-2302. https://doi.org/10.1021/ac050162j

[67] C.H. Arnaud, Open-air ionization methods minimize sample prep and widen range of mass spectrometry applications, Chemical \& Engineering News 85(2007), 13-18.

[68] Cody, R. B. Observation of molecular ions and analysis of nonpolar compounds with the direct analysis in real time ion source, Anal. Chem. 81 (2009), 1101-1107. https://doi.org/10.1021/ac8022108

[69] M. Kirchner, E. Matisová, S. Hrouzková and H. Renáta, Fast GC and GC-MS analysis of explosives, Petroleum and Coal 49 (2) (2007), 72-79.

[70] M. Yin, C. Zhang, J. Li, H. Li, Q. Deng and S. Wang, Highly sensitive detection of benzoyl peroxide based on organoboron fluorescent conjugated polymers, Polymers (Basel) 11(10) (2019), 1655. https://doi.org/10.3390/polym11101655

[71] R.G. Ewing, D.A. Atkinson, G.A. Eiceman and G.J. Ewing, A critical review of ion mobility spectrometry for the detection of explosives and explosive related compounds, Talanta 54(3) (2001), 515-529. https://doi.org/10.1016/S0039-9140(00)00565-8

[72] A.B. Kanu and H.H. Hill Jr, Identity confirmation of drugs and explosives in ion mobility spectrometry using a secondary drift gas, Talanta 73 (2007), 692-699. https://doi.org/10.1016/j.talanta.2007.04.058

[73] H. Shahraki, M. Tabrizchi and H. Farrokhpor, Detection of explosives using negative ion mobility spectrometry in air based on dopant-assisted thermal ionization, J. Hazard. Mater. 357 (2018), 1-9. https://doi.org/10.1016/j.jhazmat.2018.05.054 
[74] W. Xua, Y. Fu, Y. Gao, J. Yao, T. Fan, D. Zhu, Q. He, H. Cao and J. Cheng, A simple but highly efficient multi-formyl phenol/amine system for fluorescence detection of peroxide explosive vapor, ChemComm. (2013), 1-4. https://doi.org/10.1039/x0xx00000x

[75] J.C. Oxley, J.L. Smith, M. Porter, L. McLennan, K. Colizza, Y. Zeiri, R. Kosloff, and F. Dubnikova, Synthesis and degradation of hexamethylene triperoxide diamine (HMTD), Propellants Explos. Pyrotech. 41 (2016), 334-350.

https://doi.org/10.1002/prep.201500151

[76] J. Oxley, J. Zhang, J. Smith and E. Cioffi, Mass spectra of unlabeled and isotopically labeled hexamethylene triperoxide diamine (HMTD), Propellants Explos. Pyrotech. 25(6) (2000), 284-287. https://doi.org/10.1002/1521-4087(200012)25:6<284::AIDPREP284>3.0.CO;2-X

[77] J.M. Dreyfors, S.B. Jones, and Y. Sayed, Hexamethylenetetramine: A Review, Am. Ind. Hygiene Assoc. J. 50(1989), 579-585. https://doi.org/10.1080/15298668991375191

[78] E. Almenar, A.M. Costero, P. Gaviña, S. Gil and M. Parra, Towards the fluorogenic detection of peroxide explosives through host-guest chemistry, R. Soc. Open Sci. 5 (2018), 171787. http://dx.doi.org/10.1098/rsos.171787

[79] J. Wang, Electrochemical sensing of explosives, Electroanalysis 19 (2007), 415-423. https://doi.org/10.1002/elan.200603748

[80] C. Bauer, U. Willer, R. Lewicki, A. Pohlkötter, A. Kosterev, D. Kosynkin, F.K. Tittel and W. Schade, A Mid-infrared QEPAS sensor device for TATP detection, J. Phys. Conf. Ser. 157 (2009), 012002. https://doi.org/10.1088/1742-6596/157/1/012002)

[81] L. Widmer, S. Watson, K. Schlatter and A. Crowson, Development of an LC/MS method for the trace analysis of triacetone triperoxide (TATP), Analyst 127 (2002), 1627-1632. https://doi.org/10.1039/B208350G

[82] Y. Zhang, X. Ma, S. Zhang, C. Yang, Z. Ouyang and X. Zhang, Direct detection of explosives on solid surfaces by low temperature plasma desorption mass spectrometry, Analyst 134 (2008), 176-181. https://doi.org/10.1039/B816230A

[83] S. Girotti, E. Ferri, E. Maiolini, L. Bolelli, M. D’Elia, D. Coppe and F.S. Romolo, A quantitative chemiluminescent assay for analysis of peroxide-based explosives, Anal., Bioanal. Chem. 400 (2011), 313-320. https://doi.org/10.1007/s00216-010-4626-3

[84] M.A. Walter, U. Panne and M.G. Weller, A novel immunoreagent for the specific and sensitive detection of the explosive triacetone triperoxide (TATP), Biosensors 1 (2011), 93-106. https://doi.org/10.3390/bios1030093 
[85] E. Sella and D. Shabat, Self-immolative dendritic probe for direct detection of triacetone triperoxide, Chem. Commun. (2008), 5701-5703. https://doi.org/10.1039/b814855d

[86] M.E. Germain and M.J. Knapp, Turn-on fluorescence detection of $\mathrm{H}_{2} \mathrm{O}_{2}$ and TATP, Inorg. Chem. 47 (2008), 9748-9750. https://doi.org/10.1021/ic801317x

[87] H. Lin and K.S. Suslick, A colorimetric sensor array for detection of triacetone triperoxide vapor, J. Am. Chem. Soc. 132(44) (2010), 15519-15521. https://doi.org/10.1021/ja107419t

[88] Z. Li, W.P. Bassett, J.R. Askim and K.S. Suslick, Differentiation among peroxide explosives with an optoelectronic nose, Chem. Commun. 51 (2015), 15312-15315. https://doi.org/10.1039/C5CC06221G

[89] J.R. Askim, Z. Li, M.K. LaGasse, J.M. Rankin and K.S. Suslick, An optoelectronic nose for identification of explosives, Chem. Sci. 7 (2016), 199-206. https://doi.org/10.1039/C5SC02632F

[90] Ş. Eren, A. Üzer, Z. Can, T. Kapudan, E. Erçağ and R. Apak, Determination of peroxidebased explosives with copper(II)-neocuproine assay combined with a molecular spectroscopic sensor, Analyst 135 (2010), 2085-2091. https://doi.org/10.1039/b925653a

[91] Z. Can, A. Üzer, K. Türkekul, E. Erçağ and R. Apak, Determination of triacetone triperoxide with a N,N-Dimethyl-p-phenylenediamine sensor on nafion using $\mathrm{Fe}_{3} \mathrm{O}_{4}$ magnetic nanoparticles, Anal. Chem. 87 (2015), 9589-9594.

https://doi.org/10.1021/acs.analchem.5b01775

[92] K. Colizza, A. Yevdokimov, L. McLennan, J.L. Smith and J. C. Oxley, Reactions of organic peroxides with alcohols in atmospheric pressure chemical ionization-the pitfalls of quantifying triacetone triperoxide (TATP), J. Am. Soc. Mass Spectrom. 29 (2018), 393Y404. https://doi.org/10.1007/s13361-017-1836-3

[93] K. Colizza, K.E. Mahoney, A.V. Yevdokimov, J.L. Smith and J.C. Oxley, Acetonitrile ion suppression in atmospheric pressure ionization mass spectrometry, J. Am. Soc. Mass Spectrom. 27(2016), 1796-1804. https://doi.org/10.1007/s13361-016-1466-1

[94] D. Rondeau, R. Vogel and J.-C. Tabet, Unusual atmospheric pressure chemical ionization conditions for detection of organic peroxides, Sect. Title Org. Anal. Chem. 38 (2003), 931-940. https://doi.org/10.1002/jms.501

[95] K. Colizza, M. Porter, J.L. Smith and J.C. Oxley, Gas-phase reactions of alcohols with hexamethylene triperoxide diamine (HMTD) under atmospheric pressure chemical ionization conditions, Rapid Commun. Mass Spectrom. 29 (2014), 74-80. https://doi.org/10.1002/rcm.7084 
[96] G.A. Newsome, L.K. Ackerman and K.J. Johnson, Humidity effects on fragmentation in plasma-based ambient ionization sources, J. Am. Soc. Mass Spectrom. 27 (2016), 135143. https://doi.org/10.1007/s13361-015-1259-y

[97] T-W. Chen, Z-H. Sheng, K. Wang, F-B. Wang and X-H. Xia, Determination of explosives using electrochemically reduced graphene, Chem. Asian J. 6 (2011), 12101216. https://doi.org/10.1002/asia.201000836

[98] F. Zapata, Á. F. la Ossa, E. Gilchrist, L. Barron and C. García-Ruiz, Progressing the analysis of improvised explosive devices: Comparative study for trace detection of explosive residues in handprints by Raman spectroscopy and liquid chromatography, Talanta 161 (2016), 219-227. https://doi.org/10.1016/j.talanta.2016.05.057

[99] L. Dunn, H.S.A. Al Obaidly and S. E. Khalil, Development and validation of fast liquid chromatography high-resolution mass spectrometric (LC-APCI-QToF-MS) methods for the analysis of hexamethylene triperoxide diamine (HMTD) and triacetone triperoxide (TATP), Forensic Chemistry 10 (2018), 5-14. https://doi.org/10.1016/j.forc.2018.06.003

[100] K. Hakansson, R.V. Coorey, R.A. Zubarev, V.L. Talrose and P. Hakansson, Low-mass ions observed in plasma desorption mass spectrometry of high explosives, J. Mass Spectrom. 35 (2000), 337-346.

[101] S. Girotti, E. Ferri, E. Maiolini, L. Bolelli, M. D’Elia, D. Coppe and F.S. Romolo, A quantitative chemiluminescent assay for analysis of peroxide-based explosives, Anal. Bioanal. Chem. 400 (2011), 313-320. https://doi.org/10.1007/s00216-010-4626-3

[102] T.P. Forbes and E. Sisco, Recent advances in ambient mass spectrometry of trace explosives, The Analyst 143(9) (2018), 1948-1969. https://doi.org/10.1039/C7AN02066J

[103] D. Lubczyk, C. Siering, J. Lörgen, Z.B. Shifrina, K. Müllen and S.R. Waldvogel, Simple and sensitive online detection of triacetone triperoxide explosive, Sensors and Actuators B: Chemical. 143(2) (2010), 561-566. https://doi.org/10.1016/j.snb.2009.09.061

[104] S. Malashikhin and N.S. Finney, Fluorescent signaling based on sulfoxide profluorophores: application to the visual detection of the explosive TATP, J. Am. Chem. Soc. 130(39) (2008), 12846-12847. https://doi.org/10.1021/ja802989v

[105] X. Lü, P. Hao, G. Xie, J. Duan, L. Gao and B. Liu. A sensor array realized by a single flexible $\mathrm{TiO}_{2} / \mathrm{POMs}$ film to contactless detection of triacetone triperoxide, Sensors 19 (2019), 915 (1-12). https://doi.org/10.3390/s19040915

[106] R.S. Ray, B. Sarma, S. Mohanty and M. Misra, Theoretical and experimental study of sensing triacetone triperoxide (TATP) explosive through nanostructured $\mathrm{TiO}_{2}$ substrate, Talanta 118 (2014), 304-311. https://doi.org/ 10.1016/j.talanta.2013.09.057 
[107] S. Banerjee, S.K. Mohapatra, M. Misra and I.B. Mishra, The detection of improvised nonmilitary peroxide based explosives using a titania nanotube array sensor, Nanotechnology 20 (2009), 75502 (1-6).

https://doi.org/10.1088/0957-4484/20/7/075502

[108] X. Yu, Y. Gong, W. Xiong, M. Li, J. Zhao and Y. Che, Turn-on Fluorescent detection of hydrogen peroxide and triacetone triperoxide via enhancing interfacial interactions of a blended system, Anal. Chem. 91(11) (2019), 6967-6970.

https://doi.org/10.1021/acs.analchem.9b01255

[109] Q. Sun, Z. Wu, H. Duan and D. Jia, Detection of triacetone triperoxide (TATP) precursors with an array of sensors based on $\mathrm{MoS}_{2} / \mathrm{RGO}$ composites, Sensors 19 (2019), 1281(1-13). https://doi.org/10.3390/s19061281

[110] M.J. Song, S.W. Hwang and D. Whang, Non-enzymatic electrochemical CuO nanoflowers sensor for hydrogen peroxide detection, Talanta 80 (2010), 1648-1652. https://doi.org/10.1016/j.talanta.2009.09.061

[111] L. Luo, F. Li, L. Zhu, Z. Zhang, Y. Ding and D. Deng, Non-enzymatic hydrogen peroxide sensor based on $\mathrm{MnO}_{2}$-ordered mesoporous carbon composite modified electrode, Electrochim. Acta 77 (2012), 179-183. https://doi.org/10.1016/j.electacta.2012.05.108

[112] J-Y. Shen, M-D. Wang, Y-F. Wang, J-Y. Hu, Y. Zhu, Y.X. Zhang, Z-J. Li and H-C. Yao, Iron and carbon codoped WO3 with hierarchical walnut-like microstructure for highly sensitive and selective acetone sensor, Sens. Actuators B Chem. 256 (2018), 27-37. https://doi.org/10.1016/j.snb.2017.10.073

[113] I. Osica, G. Imamura, K. Shiba, Q. Ji, L.K. Shrestha, J.P. Hill, K.J. Kurzydlowski, G. Yoshikawa and K. Ariga, Highly networked capsular silica-porphyrin hybrid nanostructures as efficient materials for acetone vapor sensing, ACS Appl. Mater. Interfaces 9 (2017), 9945-9954. https://doi.org/10.1021/acsami.6b15680

[114] Y.J. Jeong, W.T. Koo, J.S. Jang, D.H. Kim, M.H. Kim and I.D. Kim, Nanoscale PtO catalysts-loaded $\mathrm{SnO} 2$ multichannel nanofibers toward highly sensitive acetone sensor, ACS Appl. Mater. Interfaces 10 (2018), 2016-2025.

https://doi.org/10.1021/acsami.7b16258

[115] D. Lu, A. Cagan, R.A.A. Munoz, T. Tangkuaram and J. Wang, Highly sensitive electrochemical detection of trace liquid peroxideexplosives at a Prussian-blue 'artificialperoxidase' modified electrode, Analyst 131 (2006), 1279-1281.

https://doi.org/10.1039/B613092E 
[116] R.A. Munoz, D. Lu, A. Cagan and J. Wang, One-step simplified electrochemical sensing of TATP based on its acid treatment, The Analyst 132(6) (2007), 560-565. https://doi.org/10.1039/b701356f

[117] Y. Xie and I.F. Cheng, Selective and rapid detection of triacetone triperoxide by doublestep chronoamperometry, Microchem. J. 94 (2010), 166-170. https://doi.org/10.1016/j.microc.2009.10.016

[118] V. Dobrokhotov, L. Oakes, D. Sowell, A. Larin, J. Hall, A. Kengne, P. Bakharev, G. Corti, T. Cantrell and T. Prakash, Toward the nanospring-based artificial olfactory system for trace-detection of flammable and explosive vapors, Sens. Actuators B Chem. 168 (2012), 138-148. https://doi.org/10.1016/j.snb.2012.03.074

[119] W.H. Zhang, W.D. Zhang and L.Y. Chen, Highly sensitive detection of explosive triacetone triperoxide by an $\operatorname{In}_{2} \mathrm{O}_{3}$ sensor, Nanotechnology 21 (2010), 315502. https://doi.org/10.1088/0957-4484/21/31/315502

[120] J. Warmer, P. Wagner, M.J. Schöning and P. Kaul, Detection of triacetone triperoxide using temperature cycled metal-oxide semiconductor gas sensors: Detection of triacetone triperoxide, Phys. Status Solidi A Appl. Mater. Sci. 212(2015), 1289-1298. https://doi.org/10.1002/pssa.201431882

[121] J.D. Fowler, M.J. Allen, V.C. Tung, Y. Yang, R.B. Kaner and B.H. Weiller, Practical chemical sensors from chemically derived graphene, ACS Nano 3 (2009), 301-306. https://doi.org/10.1021/nn800593m

[122] W. Yuan and G. Shi, Graphene-based gas sensors, J. Mater. Chem. A 1 (2013), 1007810091. https://doi.org/10.1039/C3TA11774J

[123] R.M. Burks and D.S. Hage, Current trends in the detection of peroxide-based explosives, Analytical and Bioanalytical Chemistry 395(2) (2009), 301-313. https://doi.org/10.1007/s00216-009-2968-5

[124] N. Bagheri, A. Khataee, J. Hassanzadeh and B. Habibi, Visual detection of peroxidebased explosives using novel mimetic Ag nanoparticle/ZnMOF nanocomposite, Journal of Hazardous Materials 360(15) (2018), 233-242.

https://doi.org/10.1016/j.jhazmat.2018.08.013

[125] X. Li, Z. Zhang and L. Tao, A novel microarray chemiluminescence method based on chromium oxide nanoparticles catalysis for indirect determination of the explosive triacetone triperoxide at the scene, Analyst 138 (2013), 1596-1600.

https://doi.org/10.1039/c3an00084b 
[126] J.C. Mbah, S. Steward and N.O. Egiebor, Solid membrane electrode assembly for on board detection of peroxides based explosives, Sensors and Actuators B: Chemical 222 (2016), 693-697. https://doi.org/10.1016/j.snb.2015.08.116

[127] R.J. Kelly, Review of safety guidelines for peroxidizable organic chemicals, American Chemical Society-Chemical Health and Safety 4(5) (1996), 33-36.

[128] M.D. Gonsalves, L. McLennan, A.L. Slitt, J.L. Smith and J.C. Oxley, In vitro metabolism of HMTD and blood stability and toxicity of peroxide explosives (TATP and HMTD) in canines and humans, Xenobiotica 51(4) (2021), 394-403. https://doi.org/10.1080/00498254.2021.1874563

[129] M.D. Gonsalves, K. Colizza, J.L. Smith and J.C. Oxley, In vitro and in vivo studies of triacetone triperoxide (TATP) metabolism in humans, Forensic Toxicol. 39 (2021), 5972. https://doi.org/10.1007/s11419-020-00540-z

[130] K. Colizza, Metabolism and gas phase reactions of peroxide explosives using atmospheric pressure ionization mass spectrometry, Open Access Dissertations, Paper 717, 2018. https://digitalcommons.uri.edu/oa_diss/717

[131] K. Colizza, M. Gonsalves, L. McLennan, J.L. Smith and J.C. Oxley, Metabolism of triacetone triperoxide (TATP) by canine cytochrome P450 2B11, Forensic Toxicol. 37 (2019), 174-185. https://doi.org/10.1007/s11419-018-0450-9 